\section{Herpes vegetans, an atypical herpes lesion in HIV patient: A case report}

\author{
Sondang P. Sirait, Wresti Indriatmi \\ Dermatovenereology Department, \\ Faculty of Medicine, Universitas; \\ Dr. Cipto Mangunkusumo General \\ Hospital, Jakarta, Indonesia
}

\begin{abstract}
Herpes vegetans is a rare form of Herpes simplex virus (HSV) infection in immunocompromised patients that clinically presents as a verrucous and hypertrophic lesion. In this case, we present a 36-year-old man with exophytic verrucous masses in the genital area that was initially suspected as a malignancy. Difficulty to properly diagnose the patient resulted in a few failed attempts at treating the lesion. After excluding other differential diagnoses, the atypical lesion proved to be caused by vegetative herpes infection due to a good response to HSV therapy. Reevaluation of biopsy also showed signs of HSV etiology. Atypical presentation of herpes simplex in immunocompromised patients still proves to be a challenge to diagnose and treat. Proper clinical identification and workup are needed to diagnose and to choose proper regiments.
\end{abstract}

\section{Introduction}

Herpes simplex virus (HSV) infection is a common infection worldwide, it is caused by 2 types of viruses. HSV Type 1 (HSV-1) infections manifest as mucocutaneous infections, mostly associated with orofacial disease. Meanwhile, HSV Type 2 (HSV-2) is usually associated with genital infections. HSV-2 infections are closely related to extensive genital lesions in different stages of evolution, including vesicles, pustules, and erythematous ulcers that require 2 to 3 weeks to resolve. ${ }^{1}$ The global prevalence of HSV-2 infections among persons aged 1449 years old is $11.9 \%$, which increased especially among patients with co-HIV infection. ${ }^{2,3}$

All manifestations of $\mathrm{HSV}$ in an immunocompetent host can be seen in an immunocompromised host, but they are more severe, more extensive, more difficult to treat, and highly recurrent. One uncommon form of chronic HSV infection, verrucous HSV or herpes vegetans, occurs both in patients with advanced AIDS and well- controlled HIV. ${ }^{1}$ In this variant, the presentation of genital herpes is atypical, which includes chronic ulcerations, papular eruptions, verrucous lesions which are hyperkeratotic, and eroded vegetating plaques that can continue for long periods. ${ }^{1,4}$ In this report, we present a case of atypical herpes lesions in HIV that initially mimics malignancy.

\section{Case Report}

A 36-year-old man presented with multiple nodules and ulcers on his glans and shaft penis, and pubic area for 4 months prior. He was referred from other dermatologist with squamous cell carcinoma based on fine needle aspiration biopsy (FNAB). The nodules began to spread and formed ulcerations. A thin white layer on top of the wound was developed. The patient was diagnosed with HIV 6 months before the examination and started antiretroviral (ARV) therapy around that period. He had been diagnosed with genital herpes before. The patient also had been taking Itraconazole for one week to treat his tinea corporis. There was a positive history of malignancy in his family, a distant relative of his had bladder cancer. On clinical examination, there were multiple erythematous plaques, nodules, and exophytic verrucous masses which were superficially eroded and covered with slough (Figure 1a). These lesions were found on the penile glans, shaft, and pubic area. The rest of the dermatological and systemic examination was normal. We suspected squamous cell carcinoma with differential diagnosis of condyloma lata, bowenoid papulosis, extramammary Paget's, and condyloma acuminata.

A $5 \mathrm{~mm}$ punch biopsy was performed, which revealed acanthotic epidermis, spongiosis, exocytosis of neutrophils and lymphocytes, and basal layer vacuolization. There were perivascular inflammatory infiltrates composed of lymphocytes, neutrophils, eosinophils, and plasma cells in the superficial and deep dermal layer. Although the histopathological presence of dense inflammatory cells and numerous plasma cells leans toward syphilis, the serologic tests (VDRL and TPHA) showed negative results. Further workup of HPV-genotyping showed positive HPV-DNA but none of the 33 subtypes $(16,18,31,33,35,39,45,51$, $52,53,56,58,59,66,68,73,81,82,6,11$, $26,40,42,43,44,54,55,57,61,70,71,72$, and 84). Based on these findings, we still cannot rule out the possibility of malignancy.

One month later, the lesions were
Correspondence: Sondang P. Sirait, Faculty of Medicine Universitas Indonesia, Salemba Raya 6, Jakarta, Indonesia.

Tel.: +62816803878

E-mail: sondangdr@yahoo.com

Key words: Herpes Vegetans, Atypical Lesion, HIV, HPV.

Contributions: WI was responsible for performing clinical examination and taking care of the patient. SPS performed biopsy, read the pathology slides, and taking care of the patient. All authors read and approved the final manuscript.

Conflict of interest: The authors declare no potential conflict of interest.

Funding: None.

Availability of data and material: Data and materials are available by the authors.

Ethical approval and informed consent: Not applicable.

Please cite this article as: Sirait SP, Indriatmi $W$. Herpes vegetans, an atypical herpes lesion in HIV patient: A case report. Dermatol Rep 2022;14:9180.

Received for publication: 11 April 2021. Accepted for publication: 21 May 2021.

This work is licensed under a Creative Commons Attribution-NonCommercial 4.0 International License (CC BY-NC 4.0).

(C) Copyright: the Author(s), 2022

Licensee PAGEPress, Italy

Dermatology Reports 2022; 14:9180

doi:10.4081/dr.2022.9180

exudative and painful. With further discussion, the diagnosis was established as atypical condiloma lata on HIV undergoing ARV therapy. The patient was given two doses of 2,4 million units benzathine penicillin injection with a week interval in between. A wet $\mathrm{NaCl} 0,9 \%$ compress was also recommended. After one week, the verrucous lesion slightly improved but was still wet and itchy.

Four months later, the patient presented again with complaints of new lesions of plaque, nodules, vesicles, and erosion covered with slough and pus at the penile shaft and glans (Figure 1b,c). In the last four months, the patient had sought a second opinion and was given podophyllin tincture and valacyclovir. The lesions dried and were excised. We reviewed the previous biopsy. The tissue showed ulceration, spongiosis, multinucleated giant cells with peripheral rimming, perivascular inflamma- 
nb-UVB phototherapy was associated. In May 2019, the psoriasis had further worsened with a PASI of 13 . We therefore decided to start a therapy with DMF, recently available in Italy, at increasing dosage as per drug data sheet.

To date, the patient is taking DMF $600 \mathrm{mg} /$ day, with good tolerability and complete clinical resolution of psoriasis.

\section{Discussion}

Chronic plaque psoriasis is the most common form of psoriasis, affecting about $90 \%$ of patients. Nowadays, as a consequence of a better understanding of the pathogenetic mechanisms of the disease, many therapeutic options have become available ${ }^{2}$. Nevertheless, finding the right therapy, especially in complex patients, can still be a challenging task. In moderate to severe psoriasis, the use of systemic therapy is recommended.

In some European countries, fumaric acid esters (FAEs) such as Fumaderm ${ }^{\circledR}$, a combination of DMF and monoethyl fumarate salts, have been used for years as a systemic therapy for psoriasis. ${ }^{3}$ In this combination, DMF is believed to be the primary active ingredient and responsible for efficacy in the treatment of psoriasis. Therefore in 2017, the European Medicines Agency (EMA) approved Skilarence ${ }^{\circledR}$, a new oral formulation of DMF for the treatment of adults with moderate-to-severe chronic plaque psoriasis in need of systemic medical therapy. ${ }^{2}$ The safety profile and efficacy of DMF were investigated by the BRIDGE trial, ${ }^{4}$ whereas other previous studies had already investigated the efficacy of FAEs in the treatment of psoriasis. ${ }^{3}$

In our patient's case, the existence of important comorbidities limiting the therapeutic range and the failure of multiple ther- apies have made the management of his psoriasis particularly complex.

Moreover, the treatment of psoriasis in patients with cancer history is especially difficult: malignancy represents at least a relative contraindication to the use of immunosuppressive drugs such as methotrexate or cyclosporine, while the use of biologics is generally considered with caution in patients that have been cancerfree for at least 5 years. ${ }^{5}$ Data in the literature appear to indicate an increased risk of certain malignancies in patients with psoriasis, especially lymphohematopoietic, head and neck, and gastrointestinal tract cancers. There is also an increased risk of nonmelanoma skin cancers (NMSC), possibly as a result of prior therapies such as p-UVA phototherapy or cyclosporine. With regard to biologic drugs, some studies suggest a possible increased risk of NMSC in subjects treated with anti-TNF alpha, while there are no reports about the other molecules. ${ }^{6}$ However, there is a lack of long-term safety data regarding newer biologic agents, which leads to a very cautious use of them in patients with a history of cancer. This also tends to cause patients to be managed with topical therapies that are less effective in moderate-severe psoriasis, with a detriment of quality of life.

In this multifaceted scenario, DMF could represent a valid therapeutic choice in terms of safety. In our experience, in fact, DMF is an effective treatment option, generally well tolerated, with few side effects that recede by adjusting the dose or by discontinuation of the drug.

\section{Conclusions}

This case is of interest because in patients with many comorbidities, there is a tendency to use only topical therapies or phototherapy even in cases of moderatesevere psoriasis, with deterioration in the patient's quality of life. Thus, DMF is a valid and safe therapeutic option in patients requiring systemic therapy either as a first line or in case of previous unsuccessful treatments.

\section{References}

1. Burlando M, Molle MF, Antonelli CT et al. Erythema multiforme after initiation of anti-interleukin-12/23 (ustekinumab) treatment for plaque psoriasis. JAAD Case Rep 2020;6:386-7.

2. Mrowietz U, Barker J, Boehncke WH et al. Clinical use of dimethyl fumarate in moderate-to-severe plaque-type psoriasis: a European expert consensus. J Eur Acad Dermatol Venereol 2018;32:3-14.

3. Blair HA. Dimethyl Fumarate: A Review in Moderate to Severe Plaque Psoriasis. Drugs 2018;78:123-30.

4. Mrowietz U, Szepietowski JC, Loewe $R$, et al. Efficacy and safety of LAS41008 (dimethyl fumarate) in adults with moderate-to-severe chronic plaque psoriasis: a randomized, doubleblind, Fumaderm ${ }^{\circledR}$ - and placebo-controlled trial (BRIDGE). Br J Dermatol 2017;176:615-23. Erratum in: $\mathrm{Br} \mathrm{J}$ Dermatol 2018;178:308.

5. Takeshita J, Grewal S, Langan SM, et al. Psoriasis and comorbid diseases: Implications for management. J Am Acad Dermatol 2017;76:393-403.

6. Elmets CA, Leonardi CL, Davis DMR, et al. Joint AAD-NPF guidelines of care for the management and treatment of psoriasis with awareness and attention to comorbidities. J Am Acad Dermatol 2019;80:1073-113. 


\section{Dimethyl fumarate as a safe and effective therapy for recalcitrant psoriasis in comorbid patients}

Martina Burlando, Mattia Fabio Molle,
Emanuele Cozzani, Aurora Parodi

Section of Dermatology, DISSAL, San Martino-IST Polyclinic Hospital, University of Genoa, Italy

\begin{abstract}
Psoriasis is a chronic condition for which multiple therapies are currently available. In particular, in cases of moderate-severe psoriasis, traditional systemic drugs or the new biological drugs can be administered. However, the treatment of patients who require systemic therapy and have multiple comorbidities can be particularly complex. Some treatment options may be in fact contraindicated or may lose effectiveness over time, reducing the options available to the dermatologists. In such circumstances, dimethyl fumarate may represent a safe and effective choice, also in patients who have already attempted biological therapies. In this regard, we report the case of a patient with moderate-severe psoriasis treated over time with various therapies (including topicals, phototherapy, traditional and biological drugs) that were discontinued due to ineffectiveness or incompatibility caused by the occurrence of concomitant diseases, who finally achieved clinical remission with dimethyl fumarate.
\end{abstract}

\section{Introduction}

Psoriasis is a chronic, immune-mediated, inflammatory disease characterized by the presence of indurated erythematous plaques covered by silvery desquamative scales that usually involve knees, elbows, trunk and scalp. ${ }^{1}$ Nowadays there are multiple therapeutic options available for the treatment of psoriasis, including topicals, phototherapy, traditional systemic and novel biologic agents. ${ }^{2}$ Systemic therapies used in severe forms of disease are based on principles with immunosuppressive or immunomodulating activity, so it can be complex in patients with multiple underlying comorbidities to find the best therapy. We present the case of a psoriatic patient with multiple pathologies and numerous previous treatments in which it was finally possible to obtain an excellent clinical con- trol of the disease thanks to the use of dimethyl fumarate (DMF).

\section{Case Report}

A 57-year-old Caucasian man has been followed at our Dermatology Clinic since 2005 for plaque psoriasis with prevalent localization to the scalp, elbows, knees, inguinal and intergluteal folds. In his medical history, the patient presented chronic hepatitis $\mathrm{C}$ virus (HCV) infection.

Until 2012, the patient experienced good clinical control of the disease through the application of topical products (steroids and vitamin D analogues) together with balneophototherapy and periodic cycles of nbUVB phototherapy.

In February 2013, the patient presented a marked clinical worsening characterized by numerous infiltrated erythematousdesquamative plaques on the trunk and limbs, with a Psoriasis Area Severity Index (PASI) score of 16. After hepatological consultation, a treatment with cyclosporine at the initial dose of $2.5 \mathrm{mg} / \mathrm{kg} /$ day was therefore started with immediate clinical response. However, after 4 months, therapy was discontinued because of an increase in serum creatinine and the patient returned to topical treatments. After several months of good clinical response, the patient had a new relapse in September 2014, with involvement of the scalp, trunk, limbs, and nail onycholysis of the hands and feet. The patient denied joint pain. At the same time, the patient tested positive to the QuantiFERON-TB Gold test and showed certain pulmonary nodules on chest X-ray. A pneumological diagnostic work-up was carried out, including bronchoalveolar lavage and microscopic and cultural examinations, which excluded the presence of Mycobacterium tuberculosis strains or malignant tumor cells. Therefore, after hepatologic consultation and the initiation of prophylactic therapy with isoniazid, the patient in February 2015 began a therapy with etanercept. At follow-up blood tests in May 2015, the patient unfortunately showed a significant increase in liver function indices so etanercept and isoniazid were discontinued, and the patient returned again to topical treatments. Meanwhile, in 2016, the patient began direct acting antiviral treatment for $\mathrm{HCV}$ with excellent response and normalization of liver function. Given the further worsening of psoriasis, etanercept in combination with topical treatments was started again in December 2016. On that occasion, the patient did not resume antituberculous prophylactic therapy due to
Correspondence: Martina Burlando, Section of Dermatology, DISSAL, San Martino-IST Polyclinic Hospital, University of Genoa, 16132 Genoa, Italy.

Tel.: +393491029766

E-mail: martinaburlando@hotmail.com

Key words: Psoriasis, Dimethyl fumarate, Comorbidities, Biologics, Malignancy.

Contributions: Study conception and design: MB, MFM, EC, AP. Acquisition of data: MB, MFM. Drafting of the manuscript: MFM. Critical revision: $\mathrm{MB}, \mathrm{EC}, \mathrm{AP}$.

Conflict of interest: The authors declare no potential conflict of interest.

Funding: None.

Ethics: All the procedures adopted in the present study were in respect to the ethical standards in the World Medical Association Declaration of Helsinki. The subject gave his written informed consent to publish the present case (including publication of images).

Please cite this article as: Burlando M, Molle MF, Cozzani E, Parodi A. Dimethyl fumarate as a safe and effective therapy for recalcitrant psoriasis in comorbid patients. Dermatol Rep 2022;14:9091.

Received for publication: 30 January 2021. Accepted for publication: 26 April 2021.

This work is licensed under a Creative Commons Attribution-NonCommercial 4.0 International License (CC BY-NC 4.0).

${ }^{\circ}$ Copyright: the Author(s), 2022

Licensee PAGEPress, Italy

Dermatology Reports 2022; 14:9091

doi:10.4081/dr.2022.9091

previous intolerance, and we opted for close follow-up visits. After an initial positive response, the patient experienced progressive secondary inefficacy so in June 2017 he was switched to adalimumab with immediate skin clearance. Unfortunately, the patient had at the same time a new progressive worsening of liver function and was listed for liver transplantation. In March 2018 , following the finding of a liver nodule suggestive of malignancy, adalimumab therapy was discontinued. In April 2018, the patient underwent liver resection with a diagnosis of hepatocarcinoma. The psoriasis relapsed again; this time poorly responsive to topical treatments. Therefore, in September 2018, a therapy with apremilast was initiated, with modest results. Given the persistence of diffuse lesions after six months of therapy (PASI 10), a new cycle of 


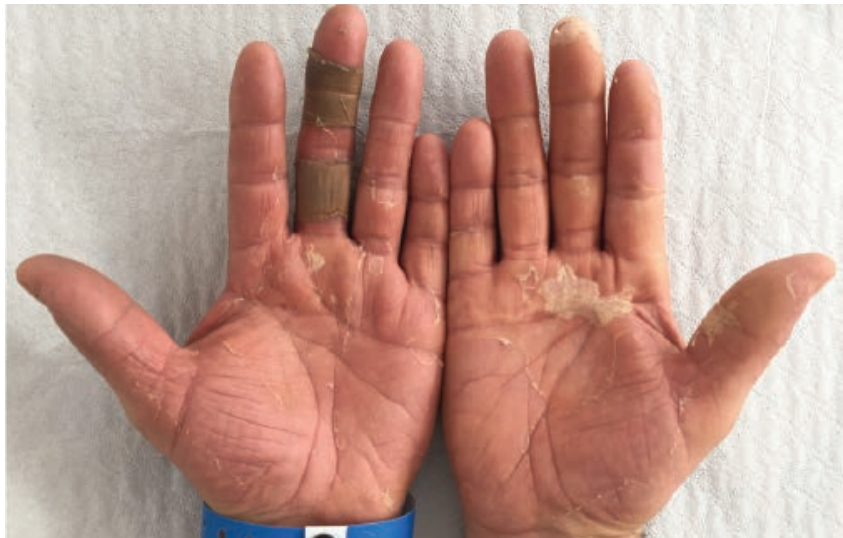

Figure 1. Exfoliative dermatitis of the palms characterized by expanding collarettes of focal peeling of the stratum corneum.

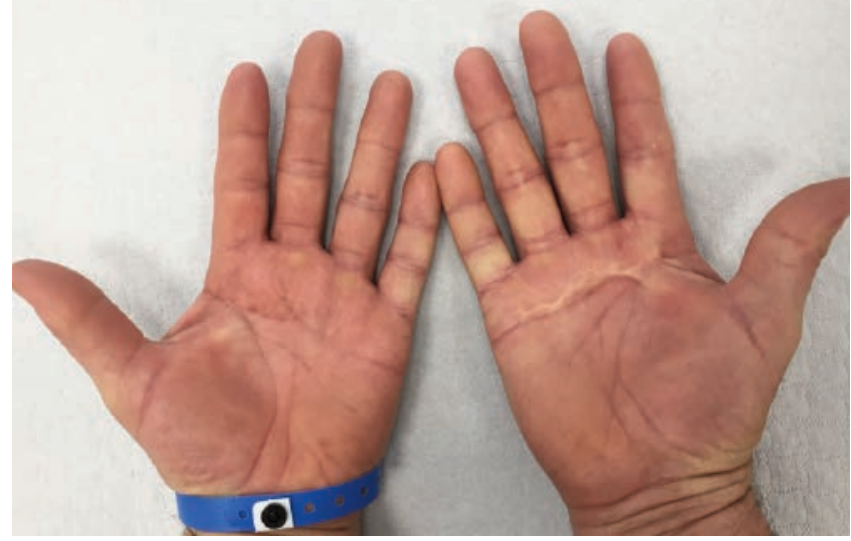

Figure 2. Complete restitutio ad integrum after suspension of cardiologic therapy with ranolazine. tion. The antianginal drug was interrupted obtaining a rapid and surprising restitutio ad integrum which allow us to confirm the diagnosis of a KE-like reaction to ranolazine. Palmoplantar exfoliation can be a consequence of genetic defects, as happens in acral peeling skin syndrome, or can be triggered by infections, as tinea pedis and scarlet fever. ${ }^{3}$ Less frequently it can be a localized manifestation of a cutaneous drug reaction, ${ }^{3}$ which may comprehend a variety of conditions that can affect acral vasomotility, hand pigmentation, skin and nails structure. ${ }^{2}$ Many chemotherapeutic agents and molecular targeted therapies can cause hand-foot syndrome, or erythrodysesthesia, which is an erythematous- desquamative and painful condition of palms and soles. ${ }^{4}$ Cardiologic therapy can rarely cause palmo-plantar desquamation and literature provides evidence of few cases triggered by calcium channel blockers, especially diltiazem, amiodarone, enalapril and phenindione. ${ }^{5}$ In our case we formulated the clinical diagnosis of $\mathrm{KE}$, also known as dyshidrosis lamellosa sicca. ${ }^{6}$ The condition is characterized by small, annular collarettes of white desquamation involving only the palms (and, less commonly, the soles) of hands and feet. ${ }^{6}$ As in our case, mechanical friction and water contact may exacerbate the cleavage and partially degradation of corneodesmosomes within the stratum corneum, the absence of an inflammatory infiltrate is characteristic. ${ }^{6}$ The discrete appearance of desquamation, limited to palms, the adult onset and the absence of inflammation signs allowed us to exclude both acral peeling skin and erythrodysesthesia. KE is a common but frequently under- recognized peeling entity with has uncertain origin, have been described both sporadic and familial cases. ${ }^{6}$ To our knowledge, literature offers evidence of only one other case of drug-induced KE, triggered by chloroquine intake. ${ }^{3}$ We herein propose the first report of a KE-like eruption to ranolazine, an antianginal molecule which selectively inhibits the late sodium current. ${ }^{7}$ Except the possibility of an immediate hypersensitivity reaction and urticaria onset, there are few evidence about ranolazine cutaneous side effects. Common side effects include dizziness, nausea and constipation while peripheral oedema, headache, asthenia, palpitations, dyspepsia, weakness and postural hypotension are less frequently reported. ${ }^{8}$

\section{Conclusions}

Mild cutaneous drug reactions are insidious and challenging diagnoses because of the high risk of mimicking other dermatological patterns that can be more frequent and probable in specific anamnestic contexts. Patient those habits can be highly suggestive for occupational dermatitis can delay the correct diagnoses for months with serious impact to their job and their social life. In our opinion, dermatologists should always keep the focus on the possibility of a drug reaction also when clinical manifestations are limited to a part of the body, as happens in fixed erythema. We suggest performing a detailed and complete pharmacological anamnesis during every medical examination as a good approach to patient in everyday clinical practice.

\section{References}

1. Sasseville D. Occupational contact dermatitis. Allergy Asthma Clin Immunol 2008;4:59-65.

2. Caccavale S, Ruocco E. Acral manifestations of systemic diseases: druginduced and infectious diseases. Clin Dermatol 2017;35:55-63.

3. Nair PA, Patel T. Palmoplantar exfoliation due to chloroquine. Indian $\mathrm{J}$ Pharmacol 2017;49:205-7.

4. Nikolaou V, Syrigos K, Saif MW. Incidence and implications of chemotherapy related hand-foot syndrome. Expert Opin Drug Saf 2016;15:1625-33.

5. Toescu SM, Kennon S, Stevens H. Diltiazem-induced palmar desquamation and oral erosions. BMJ Case Rep 2013:bcr2013201536.

6. Chang YY, van der Velden J, van der Wier G, et al. Keratolysis exfoliativa (dyshidrosis lamellosa sicca): a distinct peeling entity. Br J Dermatol 2012;167: 1076-84.

7. Rayner-Hartley E, Sedlak T. Ranolazine: a contemporary review. J Am Heart Assoc 2016;5:e003196.

8. Salazar CA, Basilio Flores JE, Veramendi Espinoza LE et al. Ranolazine for stable angina pectoris. Cochrane Database Syst Rev 2017;2:CD011747. 


\section{Keratolysis exfoliativa-like eruption induced by ranolazine}

\author{
Elena Pierobon, ${ }^{1}$ Lerica Germi, ${ }^{2}$ \\ Andrea Sechi, ${ }^{2}$ Giampaolo Trevisan, ${ }^{2}$ \\ Elena Pezzolo, ${ }^{2}$ Claudio Feliciani, ${ }^{1}$ \\ Luigi Naldi ${ }^{2}$
}

${ }^{1}$ Section of Dermatology, Department of Medicine and Surgery, University of

Parma; ${ }^{2}$ Dermatology Unit, San Bortolo

Hospital, Vicenza, Italy

\begin{abstract}
Dermatoses affecting palms may represent a dermatologic challenge from both the diagnostic, and therapeutic point of view. Patients with supposedly occupational dermatitis can spend months or even years in a frustrating attempt to avoid contact with possible irritants or allergens. To underline the importance of a thorough unbiased analysis of the patient's history and clinical features, we present the iconic case of a bricklayer affected by a chronic, disabling desquamation of palms which in the end was classified as keratolysis exfoliativa (KE) attributed to ranolazine-intake, an antianginal drug. To the best of our knowledge, this specific adverse effect of druginduced KE of palms has never been reported before in association with ranolazine.
\end{abstract}

\section{Case Report}

We report the case of a 58 old man that was referred to our Dermatological Unit presenting a 2-years history of painful desquamation of both palms of the hands. He had been working for over 20 -years as a bricklayer and the skin condition was particularly disabling interfering with ordinary life and specific job tasks.

At the dermatological examination both hands appeared affected only on the palms, showing ill-defined collarettes of dry, peeled areas with tendency to painful fissures, resembling a keratolysis exfoliativa (KE) (Figure 1).

He had no evidence of other dermatological signs or symptoms and had no previous history or hereditary predisposition for cutaneous conditions, including psoriasis and atopic dermatitis. His medical history highlighted a cardiac ischemic disease for which he had for many years a chronic therapy with antihypertensive and cholesterol-lowering medicines to which had been added 2 years before a new therapy with ranolazine to treat chronic stable angina pectoris.

Suspecting a chronic irritant contact dermatitis (ICD), we prescribed topical steroids to relieve the inflammation, suggested the use of protective gloves, barrier creams and moisturisers, providing also the appropriate information to prevent exposure to occupational irritants. The patient carefully applied our indications but at the follow up visit his dermatitis seemed to have no improvement at all. We questioned the diagnosis of ICD and focused our attention to a possible delayed-type hypersensitivity reaction. At patch testing our patient was completely negative for the most frequent haptens for general population and building works (potassium dichromate, tiuram mix and nickel sulphate).

Far from ready to give up, we underlined the temporal link from the first ranolazine intake and the onset of hand desquamation, both 2 years before. We suggest changing the cardiological therapy and after one month of ranolazine suspension, the patient's skin recovered fully (Figure 2).

\section{Discussion}

Hand dermatoses are an extremely frequent cause of dermatological consultation and may be a consequence of lots of possible agents, exogenous or endogenous, supported by irritative, allergic or infective agents. Frequently the clinical distribution and morphology of lesions are not enough specific to make the diagnosis because of a consistent risk of clinical mimicking of different aetiology. Collecting an accurate physiological anamnesis and medical history can be extremely important to investigate the role of constitutional predisposition and exogenous factors. The skin is the primary direct interface with external agents and patients those lesions affect the hands alone are likely to suffer from an occupational contact dermatitis ${ }^{1}$. Considering daily contact of our patient with wet cement, clinical features, pain and burning sensation, our case was highly suggestive for an irritant chronic eczema (ICD). ICD is caused by chemical or physical skin damage and represent approximately $80 \%$ of all cases of occupational skin disorder. ${ }^{1}$

At the failure of the first hypothesis, the second proposed diagnosis was equally possible: a minority of occupational dermatitis are allergic contact dermatitis (ACD) caused by a delayed-type hypersensitivity reaction. ${ }^{1}$ Building workers can be commonly exposed to allergens which can be detected by a properly performed patch test-
Correspondence: Elena Pierobon, Section of Dermatology, Department of Medicine and Surgery, University of Parma, Via Gramsci 14, 43126 Parma, Italy.

Tel.: +39.0521.702711 - Fax: +39.0521.702959.

E-mail: elena.pierobon@studenti.unipr.it

Key words: Keratolysis exfoliativa, Ranolazine, Cutaneous drug reaction, Hand dermatititis.

Contributions: EPi wrote most of the original draft of the paper. LG participated in drafting and designing the study. AS, EPe and GT had access to all the raw data of the study and participated in generating and gathering them. $\mathrm{LN}$ and CF revised the work critically for important intellectual content and gave their contribute in the process of analysis and interpretation of data for the work. All authors participated in writing the paper and have approved the final version.

Conflict of interest: The authors declare no potential conflict of interest.

Funding: None.

Availability of data and material: Data and materials are available by the authors.

Ethical approval and informed consent: ???

Please cite this article as: Pierobon E, Germi L, Sechi A, et al. Keratolysis exfoliativa-like eruption induced by ranolazine. Dermatol Rep 2022;14:9264.

Received for publication: 13 May 2021 Accepted for publication: 12 July 2021.

This work is licensed under a Creative Commons Attribution-NonCommercial 4.0 International License (CC BY-NC 4.0).

${ }^{\circ}$ Copyright: the Author(s), 2022

Licensee PAGEPress, Italy

Dermatology Reports 2022; 14:9264

doi:10.4081/dr.2022.9264

ing. Not at least, lesions that are clinically suggestive for an infectious aetiology, mycotic or bacterial, can be easily ruled out thanks to microscopic and culture examination. ${ }^{2}$ As these 3 most probable hypotheses came down, it is correct to focus the attention on the patient overall and reflect about the possibility of an endogenous mechanism, typical of atopic dermatitis. Although less frequent, in absence of anamnestic and clinical clues of constitutional eczema, dermatologists should consider the possibility of a drug reaction. In our case, underlying that ranolazine administration was started one month before the onset of disease, we suspected an acral drug-induced manifesta- 

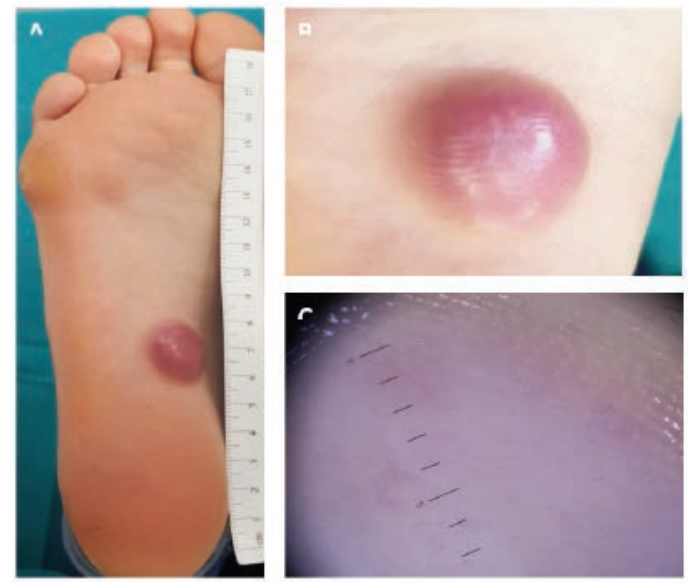

Figure 1. Clinical and dermatoscopic features of PN. A) A firm nodule of the sole of the foot of about $2 \mathrm{~cm}$ of diameter. B) Clinical detail of the well-circuscribed nodule with a homogeneous pink-purple color. C) Dermatoscopic examination didn't identify any specific vascular pattern and was featureless.
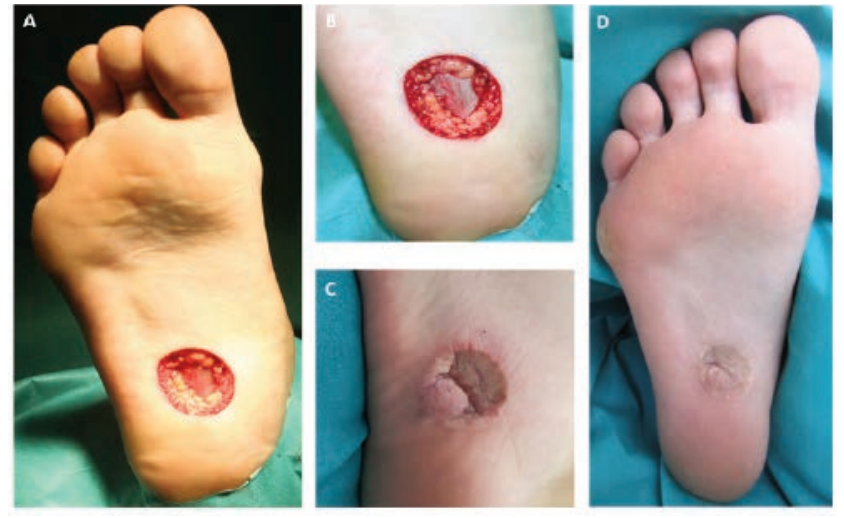

Figure 2. Surgical procedure and final outcome. A) Radical excision of the nodule of the foot with safe margins. B) Integrity of the plantar fascia ligament, at the top of which the neoplasm was located without infiltrating it. C, D) Clinical outcome of the skin graft after 3 years, perfectly healed with full recovery of the mobility function. lenging both clinically and surgically. On one hand, we would like to underline the necessity to regard some uncommon diagnoses even if the observation doesn't perfectly match all characteristics reported in literature. Extraneural $\mathrm{PN}$ is a rare entity that can be misdiagnosed because of unusual features that are not commonly reported because of the rarity of the diagnosis itself.

One the other hand, extraneural PN is a benign neoplasm with minimum risk of local recurrence and optimal prognosis after radical excision. Nevertheless, surgical removal of a tumor involving acral and functional sites could represent a challeng- ing option even when considered the treatment of choice. The consequent reconstructive procedure could be even more important than the radical excision itself and should always be performed aiming at obtaining the best functional outcome for patient.

\section{References}

1. Uerschels AK, Krogias C, Junker A et al. Modern treatment of perineuriomas: a case-series and systematic review. BMC Neurol 2020;20:1-12.
2. Rodríguez-Peralto JL, RiveiroFalkenbach E, Carrillo R. Benign cutaneous neural tumors. Semin Diagn Pathol 2013;30:45-57.

3. Hornick JL, Fletcher CDM. Soft tissue perineurioma. Am J Surg Pathol 2005;59:845-58.

4. Carter JM, Wu Y, Blessing MM et al. Recurrent genomic alterations in soft tissue perineuriomas. Am J Surg Pathol 2018;42:1708-14.

5. Yan H, Liu S, Gao W, et al. Management of degloving injuries of the foot with a defatted full-thickness skin graft. J Bone Joint Surg Am 2013;95:1675-81. 


\section{Acral perineurioma: $A$ case report of a rare neoplasm successfully treated with autologous skin graft reconstructive surgery}

\author{
Elena Pierobon, ${ }^{1}$ Chiara Cortelazzi, ${ }^{2}$ \\ Michele Maria Dominici, ${ }^{1}$ Claudio \\ Feliciani, ${ }^{1}$ Sergio Di Nuzzo' \\ ${ }^{1}$ Section of Dermatology, Department of \\ Medicine and Surgery, University of \\ Parma; ${ }^{2}$ Section of Dermatology, \\ Department of Medicine and Surgery, \\ University of Modena and Reggio \\ Emilia, Modena, Italy
}

\begin{abstract}
Soft tissues perineurioma is a rare nerve sheath tumor that affects most of all the subcutaneous tissue. Even if it could present as a large mass, it is a benign neoplasm for which a complete surgical excision represents the gold standard treatment. Considering that it usually affects acral sites of young people, it can be challenging to perform a reconstructive surgery that allows a full functional recovery. We report the case of a woman in her 20 s presenting a perineurioma of the sole of the right foot, a nodule of about $2 \mathrm{~cm}$ of diameter that compromised the support of the foot on the ground. We performed a radical surgical excision with no recurrence after 3 years of follow up and we obtained a full functional recovery thanks to an autologous full-thickness skin graft.
\end{abstract}

\section{Case Report}

A woman in her 20 s came to our attention presenting a nodular lesion of the right sole. She referred that the lesion slowly developed after a trivial trauma without any symptom. Her medical history was completely negative up to that moment and there was no evidence of familiar conditions such as neurofibromatosis. On clinical examination, we observed a single, rounded well circumscribed nodule, with a homogeneous pink-purple color and hard on palpation (Figure 1A,B). Dermatoscopic evaluation was nonspecific and featureless (Figure 1C).

The mass was painless, but the patient complained an initial discomfort with the pressure of the foot on the ground due to the size of the neoplasm, that was about $2 \mathrm{~cm}$ of diameter.

Histopathological analysis of a first incisional biopsy pointed to the diagnosis of perineurioma of soft tissues, even if the interpretation was more difficult because of the absence of the typical immunohistochemistry staining for the long cytoplasmic processes of perineurial cells (EMA).

We performed a complete surgical excision (Figure 2A), preserving the integrity of the plantar fascia ligament, that wasn't infiltrated by the neoplasm (Figure 2B). The loss of substance of the wound was reconstructed harvesting and applying an autologous full-thickness skin graft that healed successfully (Figure 2C,D).

After radical excision, our patient perfectly recovered with no recurrence after 3 years of clinical follow up.

\section{Discussion}

PN is a rare nerve sheath tumor that originates exclusively from perineural cells, which take part in the blood-nerve barrier surrounding peripheral nerves. ${ }^{1}$ Depending on the relationship with the nerve enveloped, PNs are classified into intraneural and extraneural ones, with different clinical presentation and etiology. ${ }^{2}$

As in our case, extraneural PN affects soft tissues and are mostly located in the subcutis. ${ }^{3}$ Typically, it occurs on acral sites and trunk of young to middle-aged adults as asymptomatic masses which can slowly reach a remarkable size. ${ }^{3}$ Extraneural $\mathrm{PN}$ is caused by deletions of chromosome $22 \mathrm{q}$ (with NF2 gene) or of chromosome 17q (with NF1 gene) so, as in other nerve sheath tumors, its onset can be facilitated in patients with neurofibromatosis. ${ }^{4}$

Nodules are usually yellow white, well circumscribed but unencapsulated. ${ }^{3}$ Clinical appearance is so aspecific that the diagnosis is based only on histopathological and immunohistochemical criteria. ${ }^{3}$ Microscopically we observe low cellularity ${ }^{3}$ and atypical features in about $20 \%$ of subcutaneous perineuriomas, without a clinical significance. ${ }^{2}$ Most of the tumor cells are positive for the epithelial membrane antigen (EMA) immunohistochemistry, ${ }^{3}$ that highlights the cytoplasmic processes of perineurial cells. ${ }^{2}$ EMA positivity is a useful diagnostic tool, but a small subset of tumors appears to lack it, ${ }^{3}$ as in our observation.

A complete surgical excision is the treatment of choice even if the involvement of extremities and the onset among young people can make challenging to perform a radical approach and a consequent functional reconstructive surgery. Considering the weight-bearing function of the foot, a full thickness autologous skin graft is a good
Correspondence: Elena Pierobon, Section of Dermatology, Department of Medicine and Surgery, University of Parma, Via Gramsci 14 . 43126 Parma, Italy.

Tel.: +39.0521.702711 - Fax: +39.0521.702959.

E-mail: elena.pierobon@studenti.unipr.it

Key words: Perineurioma, Full-thickness skin graft, Nerve sheath tumor, Acral neoplasm.

Contributions: EP participated collecting the data of the study and wrote the majority of the original draft of the paper. CC and MMD had access to all of the raw data of the study and participated in generating and gathering them. CF revised the work critically for important intellectual content. SdN participated in drafting and designing the study and analysis and interpretation of data for the work. All authors participated in writing the paper and have approved the final version.

Conflict of interest: The authors declare no potential conflict of interest.

Funding: None.

Availability of data and material: Data and materials are available by the authors.

Ethical approval and informed consent: ???

Please cite this article as: Pierobon E, Cortelazzi C, Dominici MM, et al. Acral perineurioma: A case report of a rare neoplasm successfully treated with autologous skin graft reconstructive surgery. Dermatol Rep 2022;14:9093.

Received for publication: 13 May 2021 Accepted for publication: 12 July 2021.

This work is licensed under a Creative Commons Attribution-NonCommercial 4.0 International License (CC BY-NC 4.0).

${ }^{\circ}$ Copyright: the Author(s), 2022

Licensee PAGEPress, Italy

Dermatology Reports 2022; 14:9093

doi:10.4081/dr.2022.9093

treatment approach because not only it acts as wound coverage but also permits to achieve stability and durability of the reconstructed site, as in our case. ${ }^{5}$

Generally, the prognosis is good: only in few cases, about 5\% in Hornick and Fletcher's observations, a local recurrence is reported, while there is no evidence of metastasis. ${ }^{3}$

\section{Conclusions}

We report this case because it is chal- 
the biological drug. Compliance with the treatment pathway, as defined by the guidelines, also brings benefits from the point of view of the economic impact on the National Health Service. In fact, the Budget Impact study analyzed how an increase in the use of Methotrexate could reduce the overall costs of patient management, generating savings in resources that the system could use to treat new patients.

In the treatment of patients with psoriasis there are important differences between Regions and even between individual Centres. An example of this is the management of waiting lists, which, being excessively long, force the patient to turn to different specialists in search of an adequate treatment, thus delaying the timing of treatment. This situation was worsened in 2020 by the recent Covid-19 pandemic, which led to further delays and greater difficulty in accessing treatment. In fact, public attention has focused on the issue of care guaranteed to non-Covid-19 patients, and it has emerged that, compared to 2019, outpatient specialist care has suffered a reduction of 18 million visits.

In addition, there is the experience of the outpatient setting, where the possibility of prescribing biological drugs depends on regional forecasts, causing a further diversification of patient management, both between regions and between local realities.

In conclusion, in order to standardize and promote proper care of patients with psoriasis, the Pso-Path Working Group agrees on the need to encourage the creation of a network that promotes collaboration and connection among all the actors involved in the overall care of the patient. In particular, it is deemed necessary that this network system be formalized in order to define the stages of the patient's diagnostic- therapeutic pathway and facilitate communication and coordination channels between hospital and territory.

Of equal importance is the need to work on increasing awareness at the territorial level, in order to avoid situations in which the patient is referred to the specialist only at an advanced stage of the pathology, delaying, even for years, both a correct diagnosis and a prescription for appropriate treatment. This situation occurs, in fact, when symptoms are not correctly identified in the early stages of the disease and the patient's progress is slowed down from the first contact. It is therefore good to insist on the importance and impact of the pathology and the possible pathways, in order to favour the possibility of recognising the symptoms at an early stage and to start the patient on an appropriate therapeutic pathway as defined by the guidelines. It is therefore desirable to formalize regional guidelines that define the points of contact within the pathway of the patient with psoriasis that take into account local specificities, set concrete goals in terms of increasing the appropriateness of care and offer shared criteria to reduce the variability of care of patients with psoriasis.

\section{References}

1. Prignano F, Rogai V, Cavallucci E, et al. Epidemiology of Psoriasis and Psoriatic Arthritis in Italy-a Systematic Review. Curr Rheumatol Rep 2018;20:43.

2. Zagni E, Colombo D, Fiocchi M, et al. Pharmaco-utilization of biologic drugs in patients affected by psoriasis, psoriatic arthritis and ankylosing spondylitis in an Italian real-world setting. Expert Rev
Pharmacoecon Outcomes Res 2020;20: 491-7.

3. Multidisciplinary Working Group in Dermatology Emilia-Romagna Region. Systemic treatment of moderate-severe chronic plaque psoriasis with particular reference to biotechnological drugs. Therapeutic guideline no.1. Update May 2019 Assessorato Cura della persona, Salute e Welfare Regione EmiliaRomagna.

4. IQVIA Holdings Inc. IQVIA data 2nd quarter 2020. Available from: https://s24.q4cdn.com/326377938/files/ doc news/2020/07/22/IQVIA-Q22020-Earnings-Press-Release_Final.pdf

5. Agenzia Italiana del Farmaco. National Observatory for medicines use. National Report 2015. Rome: Agenzia Italiana del Farmaco, 2016.

6. Zagni E, Bianchi L, Fabbrocini G, et al. A real-world economic analysis of biologic therapies for moderate-to-severe plaque psoriasis in Italy: results of the CANOVA observational longitudinal study. BMC Health Serv Res 2021;21: 924.

7. Pompilio G, Integlia D, Aru C. Analisi di impatto sul budget SSN di una maggiore appropriatezza prescrittiva in prima linea del metotrexato nei pazienti con psoriasi di grado da moderato a severo. Available form: http://clinicoeconomics.eu/articles/flipbook/16_2 021 01-12/

8. Prignano F, Tripo L, Amato L, et al. Tuscan consensus on the diagnosis, treatment and followup of moderate-tosevere psoriasis. G Ital Dermatol Venereol 2017;152:99-108.

9. 4th Salutequità Report. Missed cures in 2020. June 2021. Available from: www.salutequita.it 
monies, a subsequent update of the text had been hypothesized after 2016, the hypothesis, however, was not followed up due to the difficulty of bringing together all the indefectible stakeholders and for the related economic effort. It is precisely the need to identify shared criteria for the appropriate use of biologic drugs that led EmiliaRomagna Region to draw up new Guidelines on the Treatment of ModerateSevere Chronic Plaque Psoriasis. In May 2019, the multidisciplinary working group on Biotechnological Drugs in Dermatology of the Emilia- Romagna Region recommended the use of bDMARDs in case of non-response, intolerance or contraindication to Methotrexate and Cyclosporine. Moreover, it states that Methotrexate is the reference drug, among first-line drugs, for the treatment of moderate-to-severe chronic plaque psoriasis.

Moreover, the need to define a clinicaltherapeutic management process of the patient with psoriasis, which facilitates access to qualified services and appropriate care, while optimizing the use of healthcare resources, prompted Toscana Region to develop an ad hoc diagnostic-therapeutic protocol in 2014. This model of governance of the complexity of care and treatment aims to clarify the essential steps of patient management, from diagnosis to direct, continuous and planned follow-up. ${ }^{8}$

\section{Observations of the Pso-Path Table}

It is first of all perceived that the number of patients with psoriasis under treatment is considerably lower than what could be assumed considering the epidemiological data. This actually confirms the data available to date, according to which less than $10 \%$ of patients $(37,500)$ receive systemic treatment with conventional DMARDs or biological drugs. The possible reasons identified are several. First of all, in the reflections of the Pso-Path Table, the lack of a precise system for taking charge of the patient that defines the stages of the diagnostic-therapeutic pathway, identifies the points of contact and enables all the actors involved to be aligned and coordinated, has emerged with marked frequency. In the absence of such a definition, there is in fact the risk that the patient's pathway will be hindered by the absence of appropriate links between the territory and the hospital, leaving to the specialists the attempt to create them on the basis of their own knowledge rather than of a structured taking charge system.

This pathway appears to be slowed down right from the first contact with the general practitioner, in which the ability to correctly identify the symptoms and direct the patient, even in the early stages of the disease, towards the centre of reference is uncertain. All too often, in fact, the patient arrives at the specialist only at an advanced stage of the pathology, causing delays, even of years, both in the correct diagnosis and in the prescription of an appropriate treatment.

A further element brought to the attention of the members of the group concerns waiting lists, which are excessively long, pushing patients to turn to different specialists in search of adequate treatment. This continuous "search" and the relative movements can take months and, in this way, influence the timing of the treatment. This situation appears to be aggravated in the 2020s due to the recent Covid-19 pandemic, which has resulted in unprecedented difficulty in accessing treatment. In fact, missed treatment has become a real phenomenon that has focused public attention on the issue of care for non-Covid-19 patients. To take one example, according to a recent report, outpatient specialty care would have contracted from 2019 by 144.5 million fewer services, including more than 18 million visits. ${ }^{9}$

From the patients' point of view, the need for an in-depth examination of the different levels of compliance with the Guidelines at the level of individual regions emerged. The perception of the variability of treatment, which can compromise an appropriate treatment of the patient and the possibility of having access to adequate care, deserves a reflection on possible discriminatory situations that may occur due to the diversification of approaches between regions. The patient, in fact, who needs to be taken care of by a dedicated and customised treatment system based on his clinical situation, takes the risk, in the absence of a well-defined treatment pathway, of being started on treatment with a biological drug without previous use of Methotrexate, effectively skipping a therapeutic option that constitutes an additional opportunity for treatment.

The perception of clinicians, according to their experience, confirms the data emerging from the National Report "National Observatory on the Use of Medicines" (Osmed) compiled by AIFA in 2015 , according to which more than $77 \%$ of patients with psoriasis are started on treatment with biological drugs without a previous use of Methotrexate or Cyclosporine for at least 3 months. These results were also confirmed by the real- world study conducted by CliCon, which showed that about $77 \%$ of patients with psoriasis undergoing treatment with biologic drugs had not received a therapy based on Methotrexate or Cyclosporine for a duration of at least 3 months in the year preceding biological treatment (the results were detailed in Section 2). Therefore, in actual clinical practice there is a deviation from the current guidelines recommending the use of biological drugs in case of non-response, intolerance or contraindication to Methotrexate or Cyclosporine. In addition to this, there is the experience of the outpatient setting where the possibility of prescribing biological drugs depends on regional forecasts, causing a further diversification of patient management, both between regions and between local realities.

From the point of view of the economic impact on the NHS, a recent Budget Impact study showed how a $50 \%$ increase in the use of Methotrexate can reduce the overall costs of patient management, generating a saving of $€ 35.5$ million over a two-year period and considering a potential population of 45,560 patients. In these terms, the working group stressed the importance of prescribing firstline drugs identified by the Guidelines, including Methotrexate, since in the event of a patient not responding to treatment with a biologic, skipping therapy with Methotrexate corresponds to corresponds to a complete obliteration of a therapeutic opportunity with both clinical and economic consequences.

\section{Conclusions}

Currently it is estimated that in Italy there are about 1.8 million patients with psoriasis, of which about 500,000 with moderate-severe forms. Available data show that only 37,500 patients are under systemic treatment with conventional DMARDs or Biological drugs.

Based on its experience as clinicians, economists and patients, the Pso-Path Working Group first of all believes that compared to epidemiological data, the number of patients with psoriasis treated is small and further confirms that many patients diagnosed with psoriasis do not follow the treatment path outlined by the Guidelines with prior use of Methotrexate.

During the discussion, it was pointed out that there is a marked variability in the management of the disease and the need to identify shared criteria to ensure appropriate patient care in order to avoid possible discriminatory situations that may occur due to the diversification of approaches between regions. Moreover, compliance with the treatment pathway, as defined by the guidelines, is considered essential for the patient to benefit from Methotrexate as a therapeutic opportunity prior to the use of 
response or intolerance (therapeutic failure) to a conventional synthetic DMARD.

Studies show that about $25-30 \%$ of patients have moderate-severe forms of psoriasis $^{1}$. It follows that in Italy about 500,000 patients are affected. A recent retrospective study on the administrative databases of two Italian regions showed that out of 211,561 patients with psoriasis, $1.1 \%$ underwent therapy with biological drugs. ${ }^{2} \mathrm{~A}$ data analysis on treatments used by patients with moderate-severe psoriasis shows that only $37,500(7 \%)$ are on systemic treatment with conventional DMARDs or biologic drugs. ${ }^{4}$ At a first reflection, it is immediately evident that, compared to the assumed epidemiology, the patients treated with systemic drugs, as required by the guidelines, are quite small.

In addition, despite the indications, the treatment path of psoriasis is not in line with the guidelines.

Data emerged in 2015 from the National Report "National Observatory on the Use of Medicines" (Osmed) prepared by AIFA. In fact, it is noted that in that year the percentage of patients with psoriasis who have started treatment with biological drugs without previous use of Methotrexate or Cyclosporine for at least 3 months was $77 \% .^{5}$

The costs analysis, subject of a recent multicentre observational study, ${ }^{6}$ also deserves some reflection. In order to verify how better prescribing of Methotrexate, as expected by the Guidelines, can have an economic impact on the National Health Service, a Budget Impact study was recently conducted, showing that increasing the use of Methotrexate by $50 \%$ (from $22.05 \%$ to $33.08 \%$ ) can reduce the overall costs of patient management. Specifically, the study showed the ability to generate national savings of 35.5 million Euros over a two-year time horizon and considering a potential population of 45,560 patients. $^{7}$

The aim of this paper is to provide insights into the psoriasis patient pathway and to collect observations, criticalities and proposals for improvement from the PsoPath Working Group, of health economists, clinicians and patients. To this end, the currently available epidemiological data will be taken into consideration, reflecting on the need for their revision and then analysing the causes of the deviation of clinical practice from the current guidelines for the management of patients with psoriasis, finally proposing initiatives for improvement to avoid inappropriate prescription.

\section{Materials and methods}

\section{The Pso-Path Table}

This document is the result of a multiprofessional discussion that has as its object the analysis of the deviation of clinical practice from the current guidelines on the care of patients with psoriasis. For this purpose, a working group, called Pso-Path, was created, composed of health economists (CliCon S.r.L.) and representatives of the Confederation of Regional District Associations (CARD), of the Association of Italian Hospital DermatologistsVenereologists and Public Health (ADOI), of the International-Italian Society of Plastic- Regenerative and Oncologic Dermatology (ISPLAD), of the Italian Association of Ambulatory Dermatologists (AIDA), of the Italian Psoriatic Association Friends of the Corazza Foundation (APIAF$\mathrm{CO})$.

The Pso-Path working group, involving scientific societies and patients on the topic of psoriasis, was motivated by the common goal of identifying areas of improvement in the current diagnostic and therapeutic setting of people with psoriasis and proposing, first of all to regional institutions, models of care and therapeutic approaches that are more effective and in line with clinical guidelines.

The Working Group discussed the results of a recent study "Analisi del percorso terapeutico nei pazienti affetti da psoriasi avviati al trattamento con farmaci biologici" carried out by CliCon, whose purpose was to analyze the patterns of conventional systemic treatments prior to biological therapy in patients with psoriasis started with biological drugs, in Italian contexts of clinical practice using real-world data. The discussion of the experts also focused on the examination of the Guidelines currently available, bringing the personal contribution derived from their experience of clinical practice.

\section{Results}

\section{Analysis of clinical practice and guidelines}

The reflections of the Pso-Path have taken into consideration first of all the epidemiological data currently available. According to these, in fact, it is estimated that in Italy there are about 1.8 million patients with psoriasis, of which about 500,000 with moderate-severe forms. We then tried to understand how these patients were treated. The data available today indicate that only 37,500 patients receive systemic treatment with conventional DMARDs or biologic drugs. Hence the need to understand what mechanisms influence the non-compliance with the Guidelines in the current diagnostic and therapeutic setting of people with psoriasis.

In addition, a temporal analysis was performed in order to measure the time interval between the use of the first-line drug and the biologic, thus investigating whether the patient's pathway to treatment with biologic is attributable to the failure of the first-line treatment or to other factors.

Recently, CliCon has carried out a retrospective observational study on the services provided and in charge of the NHS for patients diagnosed with psoriasis and started on biological therapy, between January 2013 and October 2019 (inclusion period), analysing a sample of about 3.5 million assisted (details of the methodology are reported in Section 1). The results of this analysis showed a suboptimal prescriptive appropriateness for psoriasis found in real clinical practice: in fact, of the 495 patients included with psoriasis and being treated with biological drugs, only $43.2 \%(\mathrm{~N}=214)$ had been treated with Methotrexate or Cyclosporine in the year before the start of biological therapy, and of these $52.3 \%$ $(\mathrm{N}=112)$ had received conventional treatment for a period of at least 3 months. Considering the entire population included $(\mathrm{N}=495)$, only $22.6 \%$ of patients had received Methotrexate or Cyclosporine therapy for at least 3 months in the year prior to biological treatment.

It was also found that if the whole period before the index date is considered, this percentage is reduced to 18.6 (Section 2).

Data emerged from the study were the starting point for the reflections of the working group on the actual compliance of clinical practice with the guidelines. During the discussion it emerged in fact that, according to the experience of clinicians, there is a marked variability in the management of the disease and that many patients referred directly to the treatment with biological drugs, do not follow the treatment path outlined. According to the current Guidelines of the Istituto Superiore di Sanità issued in 2013, and updated in 2016, it is recommended the use of Methotrexate - as well as Cyclosporine and Acitretin - for the treatment of severe or plaque psoriasis while the recourse to the use of biological drugs should occur for patients with severe psoriasis who do not respond or have contraindications or are intolerant to systemic therapies. According to experts' testi- 


\section{Methotrexate in the therapeu- tic pathway of patients with psoriasis. Analysis of clinical practice data and comparison with guidelines}

\author{
Valeria Corazza, ${ }^{1}$ Francesco Cusano, ${ }^{2}$ \\ Ornella De Pita, ${ }^{3}$ Luigi Rossi, ${ }^{4}$ \\ G. Giovanni Virno 5 \\ ${ }^{1}$ President of the Italian Psoriatic \\ Association Friends of the Corazza \\ Foundation (APIAFCO); ${ }^{2}$ President of \\ the Association of Italian Hospital \\ Dermatologists Venereologists and \\ Public Health (ADOI); ${ }^{3}$ President of the \\ International - Italian Society of Plastic- \\ Regenerative and Oncologic \\ Dermatology (ISPLAD); ${ }^{4}$ President of \\ the Confederation of Regional District \\ Associations of Tuscany Region \\ (CARD); ${ }^{5}$ Member of the Board of \\ Directors Italian Association of \\ Ambulatory Dermatologists (AIDA), \\ Italy
}

\begin{abstract}
Psoriasis is an inflammatory skin disease with a chronic-relapsing course. It is estimated that the prevalence in Italy is $3 \%$. An adequate model of taking care of the patient with psoriasis allows the patient to benefit from the most suitable treatment option for his health needs. In this position statement the observations, criticalities and proposals for improvement of the Pso-Path Working Group, composed by health economists, clinicians and patients, on the diagnostic-therapeutic pathway of the patient with psoriasis have been collected. In particular, the deviation of clinical practice from the current Guidelines for the management of patients with psoriasis, which recommend the use of biologic drugs in case of non-response, intolerance or contraindication to Methotrexate or Cyclosporine, was evaluated. A Working Group was convened whose participants were asked to express their thoughts on the diagnostic and therapeutic pathway of the patient with psoriasis, bringing out critical elements and proposals for improvement, based on their experiences. This position statement summarizes the experiences and consensus between clinicians and patients on actions to optimize the management of patients with psoriasis undergoing biological treatment. Compared to the epidemiological data currently available, it is
\end{abstract}

believed that only a small percentage of patients with psoriasis are treated with systemic drugs. The perception of clinicians, according to their experience, confirms the data emerging from the National Report "National Observatory on the Use of Medicines" (Osmed) compiled by AIFA in 2015 , according to which more than $77 \%$ of patients with psoriasis are started to treatment with biological drugs without a previous use of Methotrexate or Cyclosporine for at least 3 months. The Pso-Path Working Group concluded that it would be desirable to incentivize, through the formalization of regional guidelines, the creation of a network system that promotes not only a greater awareness, at the territorial level, of the importance and impact of the disease and the possible paths, but also the collaboration and connection between all the actors involved in the overall care of the patient.

\section{Introduction}

Psoriasis is a chronic-recurrent inflammatory skin disease that commonly manifests itself with lesions or plaques characterized by erythema and/or desquamation. In Italy, it is estimated that about $1,800,000$ people are affected by psoriasis as the prevalence in the population is $3 \% .^{1}$ In a recent retrospective study on the administrative databases of two Italian regions, which included 8 million patients, 211,561 patients with psoriasis were identified, representing $2.6 \% .^{2}$ Moderate-severe forms affect about $1 / 4$ of patients.

The disease, because of its specifically cutaneous manifestation, which is often very visible, also has a significant impact on the quality of life of patients causing difficulties in interpersonal, social and work relationships, even reducing their selfesteem. Knees, palms of the hands, elbows, soles of the feet, scalp, genitals, trunk, are just some of the areas generally affected.

For the treatment of moderate-severe psoriasis, the use of systemic drugs is expected and, in case of ineffectiveness or intolerance, the use of biologics. However, in clinical practice the management of the pathology is rather variable.

The European guidelines "EuroGuiDerm Guideline on the systemic treatment of Psoriasis vulgaris - Part 1: treatment and monitoring recommendations - Nast et al. ", published in 2020, recommend the initiation of conventional systemic treatments as a first line of treatment, taking into account national reimbursement conditions.

In Italy, the current Guidelines of the
Correspondence: Ornella De Pità, President of the International-Italian Society of PlasticRegenerative and Oncologic Dermatology (ISPLAD), Italy.

E-mail: ornelladp@gmail.com

Key words: Psoriasis; Methotrexate; Posistion paper; Pso-Path Working Group.

Contributions: The authors contributed equally.

Conflict of interest: The authors declare no potential conflict of interest.

Funding: None.

Please cite this article as: Coraza V, Cusano $F$, De Pità $O$, et al. Methotrexate in the therapeutic pathway of patients with psoriasis. Analysis of clinical practice data and comparison with guidelines. Dermatol Rep 2022; 14:9454.

Received for publication: 28 December 2021. Accepted for publication: 28 December 2021.

This work is licensed under a Creative Commons Attribution-NonCommercial 4.0 International License (CC BY-NC 4.0).

(C) Copyright: the Author(s), 2022

Licensee PAGEPress, Italy

Dermatology Reports 2022; 14:9454

doi:10.4081/dr.2022.9454

Istituto Superiore di Sanità issued in 2013 and updated in 2016 recommend: i) the use of Methotrexate - as well as Cyclosporine and Acitretin - for the treatment of severe or plaque psoriasis; ii) the use of biologic drugs in patients with severe psoriasis who do not respond or have contraindications or are intolerant to systemic therapies.

In May 2019, in order to identify shared criteria for the appropriate use of biologic drugs, the multidisciplinary working group on Biotechnological Drugs in Dermatology of the Region of Emilia-Romagna has drawn up new Guidelines on the Treatment of Chronic Moderate-Severe Plaque Psoriasis recommending the use of bDMARDs in case of non-response, intolerance or contraindication to Methotrexate and Cyclosporine. Furthermore, it states that Methotrexate is the reference drug, among first-line drugs, for the therapy of chronic moderate-to-severe plaque psoriasis. $^{3}$

Is to be considered also the AIFA Determination No. 699 of April 15, 2019 published in G.U. No. 93 of April 19, 2019 in which it is provided that the treatment with biological drugs in NHS charge should be limited to patients with plaque psoriasis, moderate to severe grade, in case of non- 
effect of olive oil on skin barrier.

The second most common use of $\mathrm{OO}$ was for hair problems. There is limited evidence on the benefit of $\mathrm{OO}$ for hair. Oleuropein (a major component of $\mathrm{OO}$ ) has been shown to increase hair growth and hair follicle size in mice. ${ }^{14}$ One study showed that $\mathrm{OO}$ inhibited the ability of fungi to penetrate the hair follicle. ${ }^{15}$ However, OO use in specific diseases like scalp seborrheic dermatitis should be dealt with caution since its saturated and unsaturated fatty acids may stimulate the growth of Malassezia species. ${ }^{16}$ In addition, OO is used as a standard component of in vitro culture medium for Malassezia species. ${ }^{17}$

One of the limitations of our study is that it represents Riyadh city only. Thus, the results cannot be generalized to the whole country as each region has its different culture and habits. Another limitation is that shopping malls do not fairly represent the society and its different levels especially the economical level. Moreover, the lack of studies assessing knowledge, attitude, and practice related to $\mathrm{OO}$ for skin health in Saudi Arabia and worldwide, makes it difficult to guarantee the credibility of our results. This study did not record those participants who refused to participate and hence the calculation of a response rate was not possible. Furthermore, the questionnaire used for this study is a self-reported questionnaire.

\section{Conclusions}

Our study found that $88.7 \%$ of the participants have used topical OO, with the female gender being the only factor associated with $\mathrm{OO}$ use. There were various reasons for which Saudis use topical OO. The most common reasons were for skin mois- turization and hair problems. This study might serve as basis for future studies on OO use for skin health, as research on this subject is limited.

\section{References}

1. Viola P, Viola M. Virgin olive oil as a fundamental nutritional component and skin protector. Clin Dermatol 2009;27:159-65.

2. Waterman E, Lockwood B. Active components and clinical applications of olive oil. Altern Med Rev 2007;12:33142.

3. Hashmi MA, Khan A, Hanif M, et al. Traditional Uses, Phytochemistry, and Pharmacology of Olea europaea (Olive). Evid Based Complement Alternat Med. 2015;2015:541591.

4. Elolemy AT, Albedah AM. Public knowledge, attitude and practice of complementary and alternative medicine in riyadh region, saudi arabia. Oman Med J 2012;27:20-6.

5. Al-Faris EA, Al-Rowais N, Mohamed AG, et al. Prevalence and pattern of alternative medicine use: the results of a household survey. Ann Saudi Med 2008;28:4-10.

6. MacLennan AH, Wilson DH, Taylor AW. Prevalence and cost of alternative medicine in Australia. Lancet 1996;347:569-73.

7. Eisenberg DM, Davis RB, Ettner SL, et al. Trends in alternative medicine use in the United States, 1990-1997: results of a follow-up national survey. JAMA 1998;280:1569-75.

8. Fisher P, Ward A. Complementary medicine in Europe. BMJ 1994;309:107-11.

9. Ni H, Simile C, Hardy AM. Utilization of complementary and alternative medicine by United States adults: results from the 1999 national health interview survey. Med Care 2002;40:353-8.

10. Cooke A, Cork MJ, Victor S, et al. Olive Oil, Sunflower Oil or no Oil for Baby Dry Skin or Massage: A Pilot, Assessorblinded, Randomized Controlled Trial (the Oil in Baby SkincaRE OBSeRvE. Study). Acta Derm Venereol 2016;96:323-30.

11. Danby SG, AlEnezi T, Sultan A, et al. Effect of olive and sunflower seed oil on the adult skin barrier: implications for neonatal skin care. Pediatr Dermatol 2013;30:42-50.

12. Jiang SJ, Zhou XJ. Examination of the mechanism of oleic acid-induced percutaneous penetration enhancement: an ultrastructural study. Biol Pharm Bull 2003;26:66-8.

13. Katsuta Y, Iida T, Hasegawa K, et al. Function of oleic acid on epidermal barrier and calcium influx into keratinocytes is associated with N-methyl D-aspartate-type glutamate receptors. Br J Dermatol 2009;160:69-74.

14. Tong T, Kim N, Park T. Topical Application of Oleuropein Induces Anagen Hair Growth in Telogen Mouse Skin. PLoS One 2015;10:e0129578.

15. Bahuguna S, Kushwaha R. Influence of different oils on penetration of human hair by fungi. Int $\mathrm{J}$ Cosmetic Sci 1993;15:1-5.

16. Kaneko T, Makimura K, Abe M, et al. Revised culture-based system for identification of Malassezia species. J Clin Microbiol 2007;45:3737-42

17. Siegfried E, Glenn E. Use of olive oil for the treatment of seborrheic dermatitis in children. Arch Pediatr Adolesc Med 2012;166:967. 
prevalence of CAM use in the United States ranges from $32 \%$ to $54 \%{ }^{7}$ Lastly, in Europe, it has been reported to range from 20 to $50 \%{ }^{8}$ This significant difference could be due to cultural and religious differences.

The majority of our study population had a fair to excellent knowledge on the topical OO use. This could be explained by the religious background, since olive and its derivatives have been mentioned in Quran and Sunnah (Hadith). Moreover, most of the participants were familiar with the topical use of OO since $88.7 \%$ of the participants have used topical OO. The top sources of knowledge were friends/relatives and social media with only a minority who acquired knowledge from a physician. This is in agreement with Elolemy et al., who reported that mass media and family/relative were the main sources of CAM knowledge. ${ }^{4}$ This could be a result of the fact that it is more convenient to obtain medical information from social media and family members rather than from a qualified physician. Another possibility could be the reluctance of mentioning or asking about the use of CAM from patients and physicians, respectively. In multiple studies, more than half of the participants did not discuss CAM with their physicians. ${ }^{4,5}$

Females had a significantly higher attitude score as compared to males. Interestingly, female gender was the only factor associated with the use of topical OO in our study. Some studies have shown a similar trend of more use of CAM among females. ${ }^{7-9}$ This could be explained by the fact that females are more interested in cosmetics and skin care.

The most common reason for using topical OO was skin moisturization. There is conflicting evidence in the literature with regards to the effect of $\mathrm{OO}$ on skin barrier and hydration. In a pilot study, 115 neonates were divided into 3 treatment groups: no oil, OO, and sunflower seed oil. The treatments were applied twice daily on the left side of the body for 4 weeks. Both oil groups had significantly improved hydration, and no significant differences in transepidermal water loss (TEWL). However, there were some alterations in the lipid structure of the skin barrier. ${ }^{10}$ On the other hand, in a controlled study of 19 adults who applied $\mathrm{OO}$ on one forearm, $\mathrm{OO}$ resulted in significant damage to the skin barrier. ${ }^{11}$ This negative effect of $\mathrm{OO}$ on skin barrier could be attributed to a specific component of OO, which is oleic acid. According to a laboratory study on mice, oleic acid might increase the epidermal permeability. ${ }^{12}$ In addition, one study suggests that oleic acid induces inflammation of keratinocytes through $\mathrm{N} \square$ methyl $\square \mathrm{D} \square$ aspartic acid (NMDA) $\square$ type receptors. ${ }^{13}$ Moreover, NMDA receptor antagonists inhibited the increase of TEWL caused by oleic acid. ${ }^{13}$ More studies are required to determine the

Table 4. Knowledge regarding topical olive oil (OO) use.

\begin{tabular}{|c|c|c|c|c|c|c|}
\hline & Question & $\begin{array}{c}\text { Strongly } \\
\text { agree, n (\%) }\end{array}$ & $\begin{array}{l}\text { Agree, } \\
\text { n (\%) }\end{array}$ & $\begin{array}{l}\text { Neutral, } \\
\text { n (\%) }\end{array}$ & $\begin{array}{c}\text { Disagree, } \\
\text { n (\%) }\end{array}$ & $\begin{array}{c}\text { Strongly } \\
\text { disagree, } \mathrm{n}(\%)\end{array}$ \\
\hline 1 & Topical use of $\mathrm{OO}$ contributes in moisturizing the skin & $184(45.8)$ & $178(44.3)$ & $32(8)$ & $8(2)$ & $0(0)$ \\
\hline 2 & Topical 00 protects the skin from sun light & $34(8.6)$ & $63(15.9)$ & $159(40.2)$ & $119(30.1)$ & $21(5.2)$ \\
\hline 3 & Topical 00 contributes in treating skin diseases & $82(20.8)$ & $176(44.6)$ & $106(26.8)$ & $28(7.1)$ & $3(0.7)$ \\
\hline 4 & Skin lightening is one of the benefits of topical 00 & $54(13.7)$ & $110(27.8)$ & $133(33.7)$ & $85(21.5)$ & $13(3.2)$ \\
\hline 5 & Topical 00 is effective for blackheads in the skin & $27(6.8)$ & $82(20.7)$ & $191(48.2)$ & $89(22.5)$ & $7(1.8)$ \\
\hline 6 & Topical 00 limits the occurrence of wrinkles & $63(16.2)$ & $150(38.6)$ & $127(32.6)$ & $46(11.8)$ & $3(0.8)$ \\
\hline 7 & Topical 00 contributes in the regeneration of skin cells & $78(19.8)$ & $173(43.9)$ & $108(27.4)$ & $30(7.6)$ & $5(1.3)$ \\
\hline 8 & Frequent topical use of $\mathrm{OO}$ causes dryness of the skin & $21(5.3)$ & $38(9.5)$ & $95(23.9)$ & $187(47)$ & $57(14.3)$ \\
\hline 9 & Irritation of the skin in children is a disadvantage of topical 00 & $12(3)$ & $32(8.1)$ & $132(33.5)$ & $164(41.5)$ & $55(13.9)$ \\
\hline & Topical 00 delays wound healing & $19(4.9)$ & $49(12.5)$ & $178(45.5)$ & $118(30.2)$ & $27(6.9)$ \\
\hline & Topical 00 increases the risk of infection & $18(4.6)$ & $41(10.5)$ & $142(36.4)$ & $155(39.7)$ & $34(8.8)$ \\
\hline 12 & O0 has antioxidant properties & $54(13.6)$ & $137(34.6)$ & $131(33.1)$ & $63(15.9)$ & $11(2.8)$ \\
\hline 13 & OO has anti-inflammatory properties & $51(12.9)$ & $135(34.3)$ & $154(39.1)$ & $49(12.4)$ & $5(1.3)$ \\
\hline 14 & 00 contains carcinogens & $12(3)$ & $13(3.3)$ & $46(11.5)$ & $158(39.5)$ & $171(42.7)$ \\
\hline
\end{tabular}

Table 5. Attitude towards the use of olive oil (OO).

\begin{tabular}{|c|c|c|c|c|c|c|}
\hline & Question & $\begin{array}{l}\text { Strongly } \\
\text { agree, } \mathrm{n}(\%)\end{array}$ & $\begin{array}{l}\text { Agree, } \\
\text { n (\%) }\end{array}$ & $\begin{array}{l}\text { Neutral, } \\
\text { n (\%) }\end{array}$ & $\begin{array}{l}\text { Disagree, } \\
\text { n (\%) }\end{array}$ & $\begin{array}{c}\text { Strongly } \\
\text { disagree, } \mathrm{n}(\%)\end{array}$ \\
\hline 1 & Topical 00 is beneficial for skin health & $138(34.5)$ & $221(55.3)$ & $33(8.2)$ & $7(1.8)$ & $1(0.2)$ \\
\hline 2 & Topical 00 is beneficial due to its natural origin & $189(47.8)$ & $180(45.6)$ & $24(6.1)$ & $2(0.5)$ & $0(0)$ \\
\hline 3 & $\begin{array}{l}\text { Topical } 00 \text { is beneficial because it is mentioned in } \\
\text { the Quran and Sunnah }\end{array}$ & $220(55.5)$ & $144(36.5)$ & $27(6.7)$ & $2(0.5)$ & $3(0.8)$ \\
\hline 4 & $\begin{array}{l}\text { Topical } 00 \text { is beneficial because it is commonly } \\
\text { used among the society }\end{array}$ & $128(32.5)$ & $165(41.9)$ & $66(16.7)$ & $31(7.9)$ & $4(1)$ \\
\hline 5 & Topical 00 is beneficial based on personal experience & $133(34.2)$ & $171(44)$ & $59(15.1)$ & $24(6.2)$ & $2(0.5)$ \\
\hline 6 & Society's attitude towards topical 00 is positive & $165(41.2)$ & $169(42.2)$ & $43(10.7)$ & $13(3.2)$ & $11(2.7)$ \\
\hline 7 & $\begin{array}{l}\text { Topical } 00 \text { is not beneficial because I do not believe } \\
\text { in alternative medicine }\end{array}$ & $14(3.5)$ & $15(3.8)$ & $53(13.3)$ & $181(45.5)$ & $135(33.9)$ \\
\hline 8 & Downsides of using topical 00 are more than its benefits & $10(2.5)$ & $18(4.5)$ & $64(16.1)$ & $193(48.5)$ & $113(28.4)$ \\
\hline 9 & Topical 00 is not beneficial due to its lack of medical reliability & $12(3)$ & $18(4.5)$ & $64(16.2)$ & $184(46.3)$ & $119(30)$ \\
\hline
\end{tabular}


and $32(8 \%)$ had poor knowledge. The top sources of knowledge among the participants were friends/relatives (52.6\%), social media $(32.6 \%)$, and the internet $(22.5 \%)$. Only $11.4 \%$ acquired knowledge from a physician. $90.1 \%$ of the participants agreed that topical OO can help moisturize the skin. $65.4 \%$ of the participants believe that topical OO has a potential to cure skin diseases. Moreover, $47.2 \%$ stated that using topical $\mathrm{OO}$ is beneficial due to its antiinflammatory effect. Furthermore, $41.5 \%$ of the participants consider topical OO effective in skin lightening. Only $11.1 \%$ believe that topical OO can be harmful on children due to skin irritations (Table 4).

In the attitude section, the average attitude score was $4.1 \pm 0.5$ (out of 5). Females had a higher attitude score $4.2 \pm 0.5$ as compared to males $4 \pm 0.5(\mathrm{P}=0.03)$. The attitude of the participants toward topical OO use was generally positive; $89.8 \%$ of the participants agree that topical $\mathrm{OO}$ is beneficial for skin health, and $78.2 \%$ agree based on personal experience. Furthermore, $92 \%$ of the participants agree that topical OO is beneficial as it is mentioned in Quran and Sunnah. Only $7.5 \%$ agree that topical OO is not beneficial due to its lack of medical reliability (Table 5).

$51.7 \%$ of the participants did not mix $\mathrm{OO}$ with anything. The others used it with honey $(20.3 \%)$, blackseed $(17.8 \%)$, and petroleum jelly (11\%). Other mixtures included sugar, ginger, and almond oil. The majority of participants $(79.4 \%)$ started using $\mathrm{OO}$ for skin health more than 1 year ago, the other $(20.6 \%)$ started within 1 year. When asked about where do the participants purchase/obtain OO from, $46.8 \%$ obtained it from perfume stores, $38.9 \%$ from stores that specialize in selling OO products, and $32.2 \%$ from supermarkets. The most frequent quantity of $\mathrm{OO}$ used topically was 1 teaspoon $(31.5 \%)$, and less frequently 3 or more teaspoons $(25.3 \%), 2$ teaspoons (23.9\%), and $<1$ teaspoon (19.3\%). $41.8 \%$ of the participants use OO mainly before sleeping, 33.3\% after taking a shower, 33\% when symptoms become severe, $4.8 \%$ before taking a shower, and $3.7 \%$ when exposed to the sun.

\section{Discussion}

The use of OO for therapeutic and healing purposes is a well-known part of the Saudi culture. Taking into consideration the high prevalence of OO uses among Saudis and the limited evidence for its benefit in skin health, it is essential to assess the knowledge, attitude, and practice of using
OO for skin health among Saudis. In the present study, $88.7 \%$ of the participants have used topical OO. This is considered higher than any other prevalence mentioned in Saudi CAM studies. The reported preva-

lence of CAM in Saudi Arabia in two studies was $84.6 \%$ and $73 \%$, respectively. ${ }^{4,5}$ On the other hand, the prevalence of CAM use worldwide is notably less. In Australia, the prevalence of CAM use is $48.5 \% .{ }^{6}$ Also, the

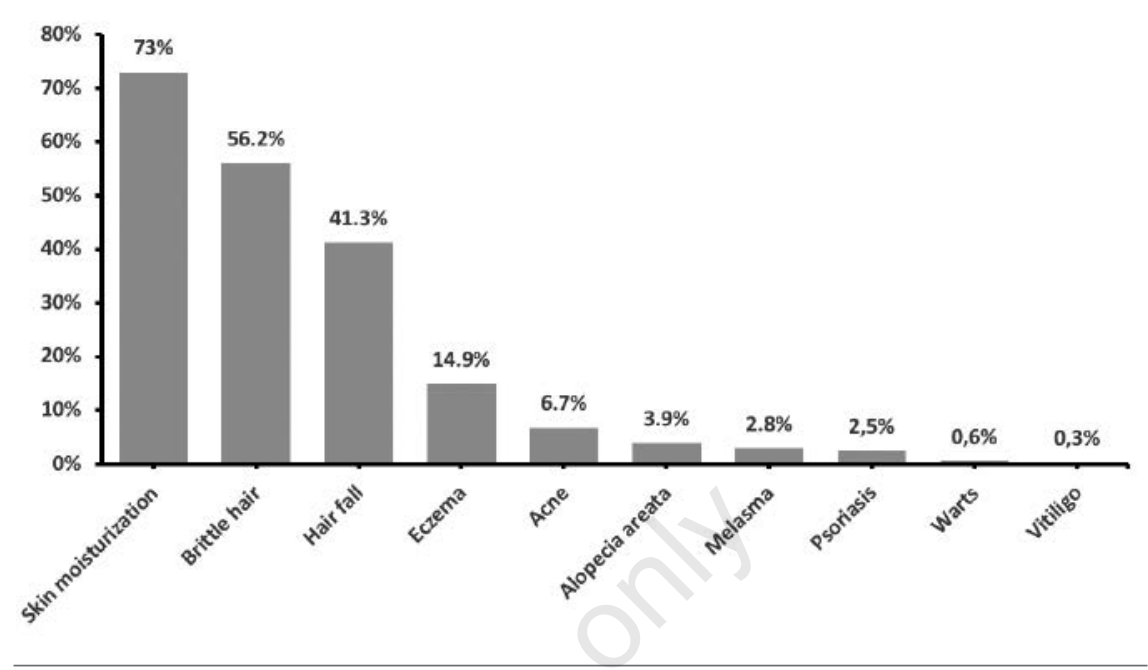

Figure 1. Conditions for which olive oil was used $(n=356)$.

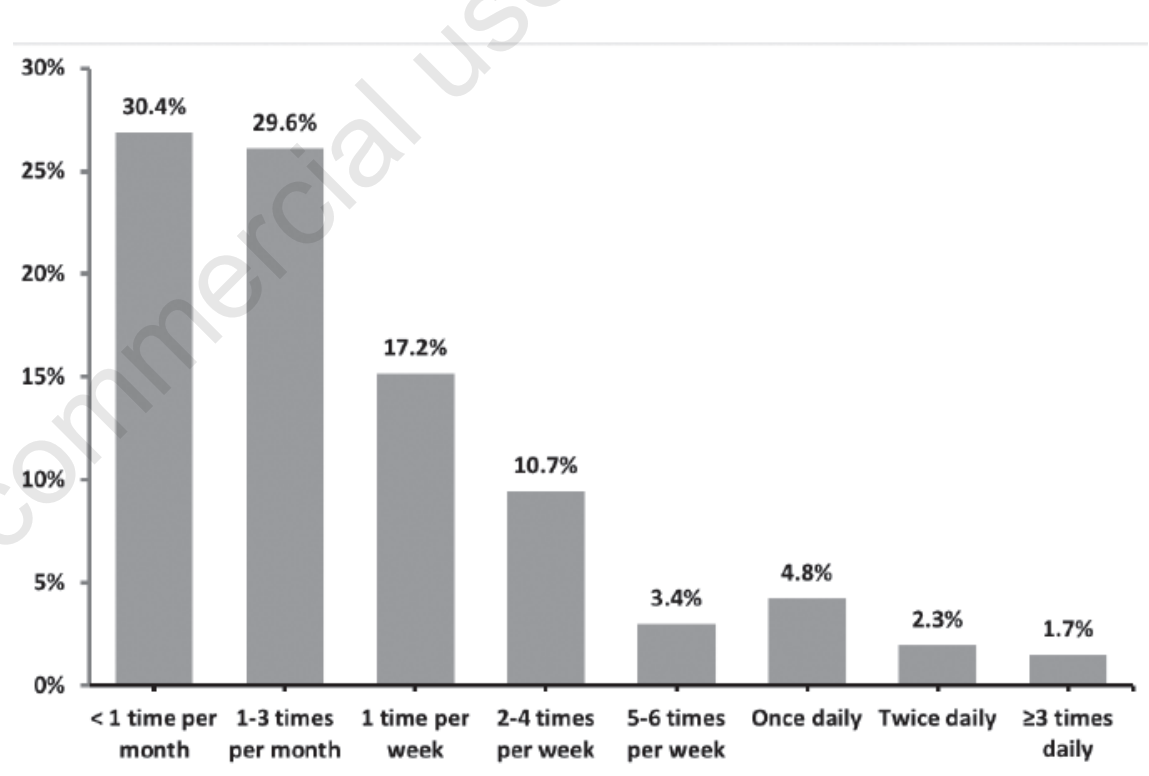

Figure 2. Frequency of topical olive oil application in the past 12 months.

Table 3. Multivariate logistic regression analysis of factors associated with the use of olive oil.

\begin{tabular}{lllcc} 
& OR & & $95 \%$ CI & P-value \\
& & Lower & Upper & \\
Female & 3.81 & 1.900 & 7.654 & $<0.001^{* *}$ \\
Income $<5,000$ SR & 1.79 & 0.759 & 4.209 & 0.184 \\
\hline Income 5,000 to 10,000 SR & 2.82 & 0.988 & 8.062 & 0.053 \\
Income $>10,000$ SR $^{*}$ & 1 & & & 0.147 \\
\hline Constant & 1.94 & & & 0.061 \\
\hline
\end{tabular}

* Reference group; **significant. 
and "strongly disagree" is equal to 5. Based on the knowledge score, the study population was categorized further into poor (1-2), fair (3-4), and excellent (5). This helps in classifying whether the majority of the population has poor, fair, or excellent knowledge.

The questionnaire was assessed by two dermatologists and one faculty member from the research unit at College of Medicine, King Saud bin Abdulaziz University for Health Sciences, for content validity. The questionnaire was then pilot tested twice. The first testing was done on 50 participants, and the second testing was done on 30 participants, to assess the clarity and time required for completion. Modifications on the questionnaire were made accordingly. The Cronbach's alpha values for both the knowledge and attitude sections were 0.674 and 0.746 , respectively.

The sample size was estimated to be 385. It was calculated with the assumption that $50 \%$ of the population use OO for skin health, with a $95 \%$ confidence level and 5\% margin of error. Convenient sampling was done, and to adjust to no responses, approximately an additional $5 \%$ were added to the sample size to become 401 .

Data analysis was done using the Statistical Package for the Social Sciences version 20 (SPSS In., Chicago, IL, USA). The descriptive statistics including frequencies and percentages were used to describe the categorical variables. The mean and standard deviation were generated for the numerical data. The univariate and multivariate logistic regression analyses were used to test the relationship between the $f$ topical $\mathrm{OO}$ and the demographics. A test with a $P$-value of $\leq 0.05$ was considered statistically significant.

\section{Results}

The total number of the participants was 401. The mean age of the participants was $27.7 \pm 8.4$ years and the majority were females (73.5\%). Of the participants, $54.5 \%$ had an education level of a bachelor's degree or higher. Moreover, $80.3 \%$ were employed and $10.3 \%$ were students (Table 1). Out of the 401 participants, $356(88.7 \%)$ have used topical OO. Moreover, 343 (96.3\%) participants who have used topical OO had a positive experience. With univariate analysis, the use of $\mathrm{OO}$ was more in females $(\mathrm{P}<0.001)$ and those with lower income $(\mathrm{P}=0.002)$ (Table 2).

Multivariate regression analysis

Table 1. Subject characteristics $(n=401)$.

\begin{tabular}{lccc}
\hline Variable & Categories & n & \\
Age (years) Mean \pm SD & & $27.7 \pm 8.4$ & \\
Gender & Male & 106 & 26.5 \\
& Female & 295 & 73.5 \\
\hline Marital status & Single & 262 & 65.3 \\
& Married & 139 & 34.7 \\
Education & Elementary & 4 & 1 \\
& Intermediate & 18 & 4.5 \\
& High school & 161 & 40 \\
& Bachelor & 205 & 51 \\
& Masters & 10 & 2.5 \\
& PhD & 4 & 1 \\
\hline Occupation & Employee & 321 & 80.3 \\
& Freelance & 9 & 2.3 \\
& Unemployed & 22 & 5.5 \\
& Student & 41 & 10.3 \\
& Other - Not salaried & 7 & 1.6 \\
Income (Saudi Riyals) & $<5000$ & 269 & 67.6 \\
& $5000-10000$ & 84 & 21.1 \\
& $10000-20000$ & 38 & 9.5 \\
& $>20000$ & 7 & 1.8 \\
\hline Olive oil use & Yes & 356 & 88.7 \\
& No & 45 & 11.3 \\
\hline & & &
\end{tabular}

showed that female gender was the only factor associated with the use of topical OO (Table 3). 67 (18.8\%) participants used topical OO for skin only, 53 (14.9\%) for hair only, and $236(66.3 \%)$ for both. The most common reason for using topical OO among the participants was skin moisturizing $(73 \%)$, brittle hair $(56.2 \%)$, and hair fall (41.3\%) (Figure 1). OO was also commonly used for eczema and acne (14.9\% and 6.7\%, respectively). $40.1 \%$ of the participants used $\mathrm{OO} \geq$ once per week over the past 12 months (Figure 2).

In the knowledge section, the average knowledge score of the participants was $3.4 \pm 0.3$ (out of 5 ). The analysis showed that $20(5 \%)$ of the participants had excellent knowledge, 349 (87\%) had fair knowledge, 


\section{The use of olive oil for skin health in a Saudi population: A cross-sectional study}

\author{
Faisal Alnemer, ${ }^{1}$ Raed Aljohani, ${ }^{1}$ \\ Abdulrahman Alajlan, ${ }^{1}$ \\ Mohammad Aljohani, ${ }^{1}$ \\ Ibrahim Alozaib, ${ }^{1}$ Emad Masuadi, ${ }^{2,3}$ \\ Aamir Omair, 2,3 \\ Mohammed I AlJasser ${ }^{1-3}$ \\ ${ }^{1}$ Division of Dermatology, College of \\ Medicine, King Saud bin Abdulaziz \\ University for Health Sciences, Riyadh; \\ ${ }^{2}$ Research Unit, College of Medicine, \\ King Saud bin Abdulaziz University for \\ Health Sciences, Riyadh; ${ }^{3}$ King \\ Abdullah International Medical \\ Research Center, Riyadh, Saudi Arabia
}

\begin{abstract}
The use of topical olive oil (OO) for skin health is common among Saudis and worldwide. Therefore, this cross-sectional study is aimed at assessing the use of topical OO for skin health among Saudis in Riyadh, Saudi Arabia. The questionnaire was administered to Saudis at four different malls in Riyadh, Saudi Arabia. A total of 401 participants were enrolled in the study. The results showed that the average knowledge score of the participants was $3.4 \pm 0.3$ (out of 5) and $87 \%$ had fair knowledge. The top source of knowledge among the participants was friends/relatives. In the attitude section, the average score was $4.1 \pm 0.5$ (out of 5). Females had a higher attitude score $4.2 \pm 0.5$ as compared to males $4 \pm 0.5$ $(\mathrm{P}=0.03)$. The most common reason for using topical OO among the participants was skin moisturizing (73\%). Female gender was the only significant factor associated with the use of topical OO $(\mathrm{P}<0.001)$. The use of topical OO for skin health is highly prevalent in Riyadh, Saudi Arabia. Therefore, more awareness of the benefits and adverse effects of topical OO use is required especially from health educational organizations. The preliminary results of this study suggest further research with a larger sample in an academic setting across the nation.
\end{abstract}

\section{Introduction}

The skin is one of the most important sites of manifestation of diseases. The consequences of poor skin health are not only limited to the physiological health, but it also extends to the psychological wellbeing. Although conventional medicine is the main treatment nowadays, complementary and alternative medicine (CAM) is becoming a popular option for the treatment of skin diseases. Olive oil (OO) is widely used among people worldwide for skin health. It is composed mainly of fatty acids and other minor components. ${ }^{1}$ The fatty acids are mainly subdivided into unsaturated (oleic and linoleic acids) and saturated types (palmitic and stearic acids). The other minor components include $\alpha$-tocopherol, phenol compounds. ${ }^{1}$ Olive oil phenols can be divided into three categories: simple phenols, secoiridoids, and lignans, all of which inhibit auto-oxidation. Major phenols include hydroxytyrosol, tyrosol, oleuropein, and ligstroside. ${ }^{2}$ This unique composition is what makes $\mathrm{OO}$ have features such as anti-inflammatory, antioxidant and antimicrobial properties. ${ }^{3}$

The studies on the use of OO for skin health are limited. The results of these studies vary from beneficial to harmful. Because of the multiple properties of $\mathrm{OO}$, it is potentially important to assess the use of OO for skin health. This might provide us with a deeper look at the significance and potentially different applications of OO. We have observed that $\mathrm{OO}$ is commonly used in Saudi Arabia; however, studies evaluating this subject are lacking. Therefore, our aim in this study is to assess the knowledge, attitude, and practice of topical OO use for skin health among Saudis in Riyadh, Saudi Arabia.

\section{Materials and methods}

The study has been performed according to the declaration of Helsinki principles and was approved by the institutional ethics committee at King Abdullah International Medical Research Center (KAIMRC) (IRBC/1576/18).

This cross-sectional study was conducted at four shopping malls during the period from February 2019 to March 2019 in Riyadh. An informed consent was obtained from all enrolled subjects. All Saudis with or without skin conditions were included in the study, while non-Saudis and illiterate people were excluded. The questionnaire was developed in Arabic and has two parts. The first part was intended to gather the demographic characteristics. These variables included gender, age, marital status, academic qualification, occupation, income, and source of knowledge. The second part of the questionnaire consisted of 33 questions divided into three sections which were the knowledge (14 questions),
Correspondence: Faisal Khalid Alnemer, Division of Dermatology, College of Medicine, King Saud bin Abdulaziz University for Health Sciences, P.O. Box 3660, Riyadh 11481, Saudi Arabia.

E-mail: Alnemer467@ksau-hs.edu.sa

Key words: Olive oil; Complementary and alternative medicine; Skin; Dermatology.

Contributions: The authors contributed equally.

Conflict of interest: The authors declare no potential conflict of interest.

Funding: None.

Ethical approval: Reviewed and approved by the institutional ethics committee at King Abdullah International Medical Research Center (IRBC/1576/18).

Availability of data and material: Data and materials are available by the authors.

Please cite this article as: Alnemer F, Aljohani $R$, Alajlan A, et al. The use of olive oil for skin health in a Saudi population: A cross-sectional study. Dermatol Rep 2022;14:9364.

Received for publication: 25 August 2021. Accepted for publication: 4 September 2021.

This work is licensed under a Creative Commons Attribution-NonCommercial 4.0 International License (CC BY-NC 4.0).

(C) Copyright: the Author(s), 2022

Licensee PAGEPress, Italy

Dermatology Reports 2022; 14:9364

doi:10.4081/dr.2022.9364

attitude (9 questions), and practice (10 questions). The knowledge and attitude questions were scored using the five-point Likert scale ranging from "strongly agree" to "strongly disagree". To formulate both the average knowledge and attitude scores, the five-point Likert scale values were used ranging from 1-5. The average score for each participant was calculated by adding up the score of every question and dividing by the number of questions. Furthermore, to calculate the average scores for the whole study population, the average scores for every participant in each section were summed up and divided by the number of participants. To make sure that the questionnaire is answered carefully and diligently, negative questions have been added to the knowledge section. Questions 2, 10, 11, 14 are presented as wrong statements about $\mathrm{OO}$ and its use. The five-point Likert scale values in these questions are reversed, which means "strongly agree" is equal to 1 
ties. ${ }^{10,14,17}$ We also established the comparable result that $\mathrm{H}$ and $\mathrm{M}$ area were associated with nodular subtype, whereas L area was related to superficial BCC. ${ }^{14}$

Considering younger age group, superficial BCC is found to be the most common BCC subtype. ${ }^{14,17}$ Furthermore, a study from Canada reports that superficial BCC is more common in women. ${ }^{18}$ However, there were no significant association between age group, sex and histological subtype in our study. There were some limitations in the current study. First, this was an academic setting, single center study which may not be the representative of whole Thai populations. Second, the pathology reports were conducted by different pathologists from both inside and outside the institute, which may affect the histological classification of the tumors. Third, because of the retrospective nature, patient modifiers such as some environmental and behavioral factors were not recorded, thus their association with the tumors could not be established. Further large multicenter study or nationwide collaboration would be appreciated.

\section{Conclusions}

In conclusion, $\mathrm{BCC}$ is prominently occurred in elderly people. Pigmented BCC is predominated in Asians. Nodular BCC is the most common histological subtype. There is a significant association between histological subtype and anatomical location, as nodular subtype is associated with $\mathrm{H}$ and $\mathrm{M}$ area, while superficial BCC was linked to L location. Quarter of the patients have the long history of large tumors before seeking medical attention. Patient's education about skin cancer may help to improve this situation. The clinical misdiagnosis of $\mathrm{BCC}$ was not unusual; therefore, the clinicians should consider BCC as one of the differential diagnosis of pigmented skin conditions in Asian populations.

\section{References}

1. Asgari MM, Moffet HH, Ray GT, et al. Trends in basal cell carcinoma incidence and identification of high-risk subgroups, 1998-2012. JAMA Dermatol 2015;151:976-81.

2. Lomas A, Leonardi-Bee J, Bath-Hextall F. A systematic review of worldwide incidence of nonmelanoma skin cancer. Br J Dematol 2012;166:1069-80.

3. Leiter U, Keim U, Garbe C. Epidemiology of skin cancer: Update 2019. Adv Exp Med Biol 2020;1268: 123-39.

4. Eisemann N, Waldmann A, Geller AC, et al. Non-melanoma skin cancer incidence and impact of skin cancer screening on incidence. J Invest Dermatol 2014;134:43-50.

5. Ciążyńska M, Narbutt J, Woźniacka A, Lesiak A. Trends in basal cell carcinoma incidence rates: a 16-year retrospective study of a population in central Poland. Adv Dermatol Allergol 2018;35:47-52.

6. Loh TY, Ortiz A, Goldenberg A, Brian Jiang SI. Prevalence and clinical characteristics of nonmelanoma skin cancers among Hispanic and Asian patients compared with white patients in the United States: A 5-year, single-institution retrospective review. Dermatol Surg 2016;42:639-45.

7. Kikuchi A, Shimizu H, Nishikawa T. Clinical and histopathological characteristics of basal cell carcinoma in Japanese patients. Arch Dermatol 1996;132:320-4.

8. Imsamran W, Pattatang A, Supaattagorn $\mathrm{P}$, et al., editors. Cancer in Thailand Vol.IX, 2013-2015. Bangkok: New Thammada Press; 2018.

9. National Cancer Institute, Department of Medical Services, Ministry of Public Health, Thailand. Hospital-based cancer registry annual report 2014. Bangkok: Pornsup Printing; 2016.
10. Puavilai S, Sirapan S. Correlation of histological subtypes of basal cell carcinoma with age, sex and distribution of skin lesions: a five-year study at Ramathibodi Hospital. J Med Assoc Thai 2002;85:560-4.

11. Bichakjian CK, Olencki T, Aasi SZ, et al. Basal Cell Skin Cancer, Version 1.2016, NCCN Clinical Practice Guidelines in Oncology. J Natl Compr Canc Netw 2016;14:574-97.

12. Sng J, Koh D, Siong WC, Choo TB. Skin cancer trends among Asians living in Singapore from 1968 to 2006. J Am Acad Drmatol 2009;61:426-32.

13. Perera E, Gnaneswaran N, Staines C, et al. Incidence and prevalence of nonmelanoma skin cancer in Australia: A systematic review. Australas J Dermatol 2015;56:258-67.

14. Cameron MC, Lee E, Hibler BP, et al. Basal cell carcinoma epidemiology; pathophysiology; clinical and histological subtypes; and disease associations. J Am Acad Dermatol 2019;80:303-17.

15. Cheng SY, Luk NM, Chong LY. Special features of non-melanoma skin cancer in Hong Kong Chinese patients: 10-year retrospective study. Hong Kong Med J 2001;7:22-28.

16. Yélamos O, Braun RP, Liopyris K, et al. Usefulness of dermoscopy to improve the clinical and histopathological diagnosis of skin cancers. J Am Acad Dermatol 2019;80:365-77.

17. Scrivener Y, Grosshans E, Cribier B. Variations of basal cell carcinomas according to gender, age, location and histopathological subtype. $\mathrm{Br} \mathrm{J}$ Dermatol 2002;147:41-7.

18. Rivers JK, Mistry BD, Hung T, et al. A 13-year retrospective study of basal cell carcinoma in a Canadian practice: a comparison between anatomical location and histopathological subtypes. J Cutan Med Surg 2016;20:233-240. 
(52.5\%), 98 (35.3\%) and 34 (12.2\%) BCCs were located on $\mathrm{H}, \mathrm{M}$ and $\mathrm{L}$ area, respectively. The most common site of distribution was the nose ( 80 cases, $28.8 \%$ ), followed by cheek (68 cases, $24.5 \%$ ), periorbital area (27 cases, 9.7\%), trunk ( 20 cases, $7.2 \%$ ) and forehead (19 cases, 6.8\%). From all, $172(61.9 \%)$ were pigmented lesions, which totally occurring in Asians, and 121 (43.5\%) BCCs were ulcerated. Most of the BCC tumors $(60.2 \%)$ were less than $10 \mathrm{~mm}$ in size, but $14.3 \%$ of them were bigger than $20 \mathrm{~mm}$. The initial clinical diagnosis was made correctly before performing the skin biopsy in 225 cases $(80.9 \%)$. Whereas the rest $(19.1 \%)$, presented in Figure 3, were misdiagnosed as melanocytic nevus, seborrheic keratosis, Bowen's disease, actinic keratosis, squamous cell carcinoma, melanoma and others. Regarding 53 misdiagnoses, 14 cases $(26.4 \%)$ were evaluated by board-certified dermatologists and 39 cases $(73.6 \%)$ were assessed by the trainees: dermatology residents or postgraduate diploma students.

The distribution of histological subtypes showed the predominance of the nodular BCC (176 cases, 63.3\%), followed by mixed (48 cases, $17.3 \%$ ) and superficial subtype (36 cases, 12.9\%). According to Table 1, a significant association between anatomical distribution and histological subtype was revealed in the study. $\mathrm{H}$ area $(\mathrm{OR}=5.03$, 95\% CI 2.31-12.29, P-value $<0.001)$ and $\mathrm{M}$ area $(\mathrm{OR}=7.30,95 \% \mathrm{CI}$ 3.03-17.64, P-value $<0.001$ ) were related to nodular BCC, while L area was linked to superficial BCC. Association between pigmented lesion and nodular type was found $(\mathrm{OR}=1.99$, 95\% CI 1.20-3.31, P-value $<0.001)$. There was no statistically significant association between sex, age group and the histological subtype $(\mathrm{P}>0.05)$.

Most of the patients $(84.5 \%)$ were surgically treated, though 18 patients $(6.5 \%)$ got non-surgical modalities (cryosurgery, electrodesiccation and curettage, photodynamic therapy and/or topical medications). The majority of the tumors $(87.7 \%)$ were completely cured, 2 patients $(0.7 \%)$ who obtained non-surgical treatment had tumor recurrence and the rest were referred to other hospitals. No metastasis was seen.

\section{Discussion}

Based on formerly published data, the incidence of NMSC in Asians is low when compared with populations in Australia, US and western countries. However, there is approximately $10 \%$ per year rise in the incidence of BCC worldwide. ${ }^{1,2,12,13}$ This ascending trend was also noticed in our study until 2016, since after that year the hospital building was under renovation which could affect the patients' accessibility. Most of the patients in the present study were older than 60 years old. Head and neck area was the most common location of BCC tumors. In addition, relatively female predominance was observed in the study. These findings were similar to the prior research reporting majority of older age group and head and neck region in overall BCC patients. ${ }^{1,6}$ Though, female gender predilection is noted only in Hispanic and Asians. ${ }^{6}$

BCC typically runs a gradually progressive course; some tumors may grow at a very slow rate and behave like benign conditions. ${ }^{14}$ This could be a possible explanation that a quarter of the patients in current study had the tumors for longer than 5 years before they started seeking medical care. Also, quarter of them already had very large tumors (greater than 20-mm in size) at their first presentation. Patient's education about skin cancer self-examination may help to improve this problem in Thailand.

Pigmented BCC was observed in 61.9\% of the patients in this study and was significantly correlated with nodular histological subtype. The findings were consistent with previous observations accounting that pigmented BCC has obviously higher prevalence in Asians, some describe the clinical appearance of brown or black pigmentation, especially at the border of the lesions. ${ }^{6,7,15}$ However pigmented BCC is a clinical and histological term, it could be microscopically superficial or nodular histological subtypes containing melanin. ${ }^{14}$ According to their dark colors, pigmented BCC had sometimes been misdiagnosed as other pigmented skin conditions. Therefore, it is very important for clinicians to consider BCC as one of the differential diagnosis in Asian patients. ${ }^{6}$ In the present study, we found misdiagnosis of BCC in $19.1 \%$ of all cases, surprisingly about one-fourth (26.4\%) were made by board-certified dermatologists. The lesions were mostly misdiagnosed as benign and premalignant cutaneous tumors including melanocytic nevus, seborrheic keratosis, Bowen's disease and actinic keratosis. In order to improve this issue, the dermoscope may play an essential role. As some clues from dermoscope such as leaflike structures, blue ovoid nest, arborizing vessels and ulceration can enhance the sensitivity and specificity for BCC detection. ${ }^{16}$ From current study, nodular BCC was the most common histological subtype. This finding was in concordance with previous studies from many countries. .,14,17 $^{-1}$ Besides, previous researches identify a significant association between the histopathologic subtypes and anatomical location, with nodular BCC being more common on chronically sun-exposed area (e.g. head and neck), while superficial BCCs were more likely to be located on trunk and extremi-

Figure 3. Provisional diagnosis other than basal cell carcinoma $(n=53)$. 
guideline, the anatomical location of BCC in the study was classified into 3 groups including $\mathrm{H}$ (central face, eyelids, eyebrows, periorbital, nose, lips, chin, mandible, pre- and postauricular, ear, temple, genitalia, hands and feet), M (forehead, cheeks, scalp, neck and pretibia) and $\mathrm{L}$ area (trunk and extremities, excluding hands, feet, nails, pretibial and ankles). ${ }^{11}$

Descriptive statistics were used to describe the demographic, clinical and histopathological data. The percentage distribution of BCC cases was calculated according to the variables. The associations of the variables were analyzed by the Pearson $\chi^{2}$ test. Multiple logistic regression analysis was performed to test the association among multiple variables and histologic subtype simultaneously. All statistical analyses were done using SPSS Statistics 23.0 (IBM corporation, Armonk, NY, USA). The P-value $<0.05$ was considered statistically significant.

\section{Results}

From a total of 901 new BCC cases diagnosed between January 2009 and December 2018, 278 cases were enrolled to the study. The trend of number of cases each consecutive year was demonstrated in Figure 1. The male to female ratio was 0.73 (161 females and 117 males). The patients were classified into three age groups: $<40$, $40-60$ and $>60$ years of age. Most of them $(71.6 \%)$ were more than 60 years old, as shown in Figure 2. Nearly all of the patients $(88.8 \%)$ were Thais and $41.0 \%$ of all were living in Bangkok.

About half $(50.6 \%)$ of the patients had the tumor for less than 2 years, whereas $16.9 \%$ of them had the lesion for longer than 5 years before seeking medical attention $($ mean $\pm \mathrm{SD}=3.10 \pm 4.53$ years $)$. Concerning anatomical location, 146

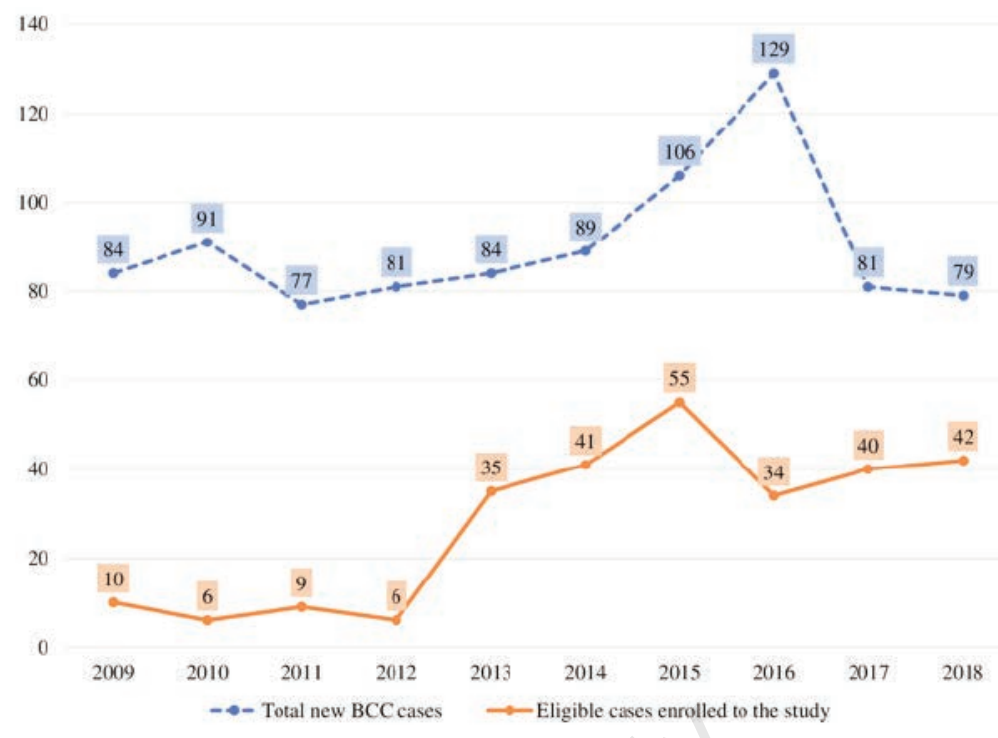

Figure 1. The annual number of total new basal cell carcinoma cases and eligible cases enrolled to the study during 10 -year study period.

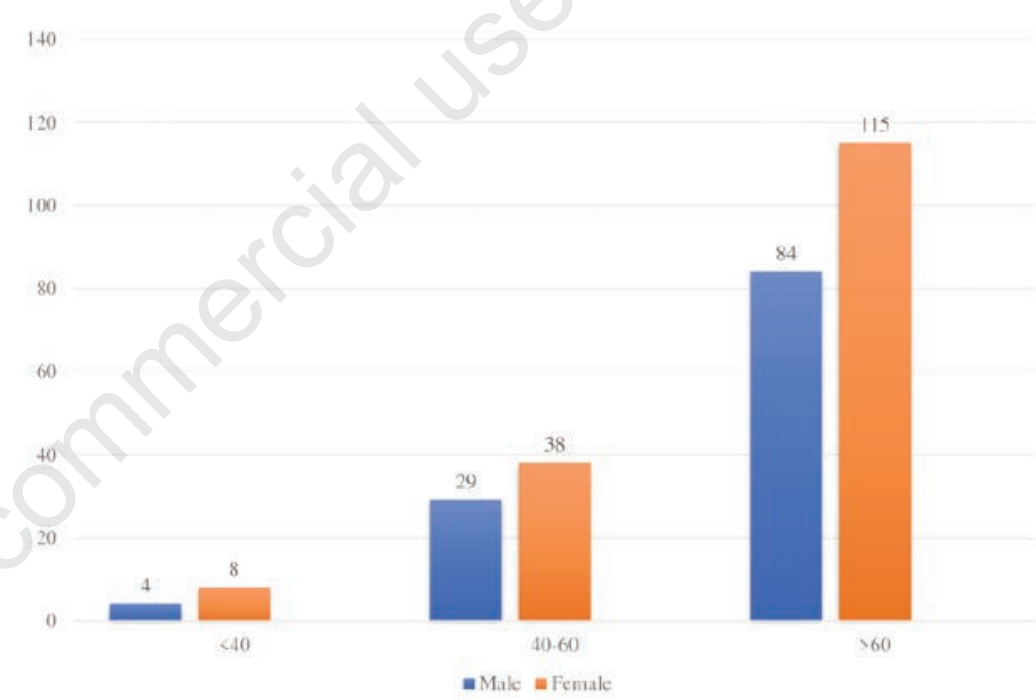

Figure 2. Age distribution of basal cell carcinoma patients $(\mathrm{n}=278)$.

Table 1. Multiple logistic regression analysis of patients' sex, age group, tumor's anatomical location related to basal cell carcinoma histological subtype.

\begin{tabular}{|c|c|c|c|}
\hline \multirow[t]{2}{*}{ Variables } & \multicolumn{3}{|c|}{ Histological subtype of basal cell carcinoma - OR $(95 \% \mathrm{CI})$} \\
\hline & Nodular subtype & Superficial subtype & Others \\
\hline \multicolumn{4}{|l|}{ Sex } \\
\hline Male & $0.94(0.56-1.55)$ & $1.53(0.73-3.21)$ & $0.84(0.48-1.46)$ \\
\hline Female & $1.06(0.65-1.74)$ & $0.65(0.31-1.37)$ & $1.20(0.69-2.10)$ \\
\hline \multicolumn{4}{|c|}{ Age group (years) } \\
\hline$>40$ & 1 & 1 & 1 \\
\hline $40-60$ & $1.06(0.30-3.68)$ & $1.50(0.17-13.15)$ & $0.80(0.21-2.94)$ \\
\hline$>60$ & $1.31(0.40-4.30)$ & $1.73(0.21-13.92)$ & $0.55(0.16-1.92)$ \\
\hline \multicolumn{4}{|c|}{ Anatomical location of tumor } \\
\hline L area & 1 & 1 & 1 \\
\hline M area & $7.30(3.03-17.64)^{*}$ & $0.05(0.02-0.15)^{*}$ & $1.27(0.47-3.48)$ \\
\hline H area & $5.03(2.31-12.29) *$ & $0.06(0.03-0.16) *$ & $1.70(0.66-4.42)$ \\
\hline
\end{tabular}




\section{Basal cell carcinoma trends in Thailand: A 10-year retrospec- tive study of demographic, clinical and histopathological features}

\author{
Anakaporn Tiyawatanaroj, Poonnawis \\ Sudtikoonaseth, Onjuta Chayangsu \\ Institute of Dermatology, Bangkok, \\ Thailand
}

\begin{abstract}
Basal cell carcinoma (BCC) is the most common skin cancer with globally increasing incidence. To date, the information regarding BCC in Thailand is limited. Our aim was to evaluate the demographic, clinical, histopathological trends of BCC and other contributing factors. We retrospectively reviewed the demographic, clinical and histological data of all BCC outpatients from January 2009 to December 2018. From 278 BCC patients recruited to this study, most of them $(71.6 \%)$ were older than 60 years old. The most common histological subtype was nodular BCC (63.3\%). A statistically significant association was observed between histological variant and location of the tumor; $\mathrm{H}$ and $\mathrm{M}$ area were associated with nodular BCC; L area was related to superficial subtype $(\mathrm{P}<0.001)$. Misdiagnosis of BCC was observed in 53 cases, mostly as melanocytic nevus $(30.2 \%)$, and about quarter $(26.4 \%)$ was made by board-certified dermatologists. Nodular BCC is significantly associated with $\mathrm{H}$ and $\mathrm{M}$ area, while superficial subtype is related to L location. Quarter of the patients already have large tumors at their first presentation. Misdiagnosis of $\mathrm{BCC}$ is not uncommon.
\end{abstract}

\section{Introduction}

Non-melanoma skin cancer (NMSC), comprising of basal cell carcinoma (BCC) and squamous cell carcinoma (SCC), is the most commonly occurring malignant tumor in white populations. BCC accounts for most $(80 \%)$ of it. ${ }^{1}$ Despite the difficulties in capturing and registering data leading to underreporting of $\mathrm{BCC}$, an upward trend in overall incidence has been noted. ${ }^{1,2}$ The incidence rate of $\mathrm{BCC}$ in United States(US) arose approximately $18 \%$ during 15-year period up to 535 cases per 100,000 personyears in 2012. ${ }^{1}$ This may be linked to the rising of aging population, sun-seeking behavior and recent advance in diagnostic technology. ${ }^{1,2}$ Although metastasis and mortality from $\mathrm{BCC}$ is rare, it can be locally destructive causing morbidity and significant burden in healthcare system. ${ }^{3-5}$

For Asian patients, BCC lesions frequently develop on sun-exposed area of head and neck region like in Caucasians. ${ }^{6}$ However, female predominance and high prevalence of pigmented variant, described as black pearly appearance, are notably observed in Asians. ${ }^{6,7}$

In Thailand, the age-specific incidence rate of skin cancer is 3.6 and 3.8 per 100,000 person-years for male and female respectively during the year 2013-2015. ${ }^{8}$ In spite of its small incidence compared with those western countries, skin cancer is not uncommon since it is one of the top fifteen leading site of new cancer patients in Thailand. $^{9}$ Moreover, the correlation between anatomical distribution and particular histological subtypes are documented in previous study from Thailand, as superficial $\mathrm{BCC}$ is predominantly occurred on the trunk, while other subtypes are more common on the head and neck. ${ }^{10}$ So far, the data regarding $\mathrm{BCC}$ in Thailand are still restricted, as long as some are in Thai language and some are unsearchable via major online medical databases and most of which are not up to date.

The aim of the present study was to analyze the epidemiologic, clinical, and histological characteristics of BCC in Thailand for 10 years and other associating variables.

\section{Materials and methods}

This study was approved by the Ethics Committee of the Institute of Dermatology, Bangkok, Thailand (IRB/IEB 022/2562) and was performed in accordance with the World Medical Association's Helsinki Declaration and its amendment.

A single-center, 10-year retrospective descriptive study was performed by medical records review of outpatients presented at Institute of Dermatology, Bangkok, Thailand from January 2009 to December 2018. There were 901 new cases that were diagnosed as primary $\mathrm{BCC}$ during the period. After excluding the tumors diagnosed without pathology reports and without or unclassified histological subtypes, 278 eligible cases were recruited to the study. Patients' demographic data, the duration of tumor's presence, past medical history, cutaneous findings, provisional clinical diagnosis, histological subtypes, treatment regimen and the result were collected.

According to the National Comprehensive Cancer Network (NCCN)
Correspondence: Onjuta Chayangsu, Institute of Dermatology 420/7 Ratchawithi Rd, Thung Phata Thai, Ratchatewi, Bangkok, 10400 Thailand. E-mail: onjuta@gmail.com

Key words: Basal cell carcinoma, skin cancer, non-melanoma skin cancer, histopathology, demography, epidemiology

Acknowledgment: The authors are grateful to Assoc. Prof. Dr. Jaranit Kaewkungwal from Faculty of Tropical Medicine, Mahidol University for his statistical advice.

Contributions: AT contributes to the acquisition, analysis, interpretation of the data, drafting the work, final approval of the version to be published and agreement to be accountable for all aspects of the work. PS contributes to the conception and design of the work, revising the manuscript critically for important intellectual content, final approval of the version to be published and agreement to be accountable for all aspects of the work. OC contributes to the conception or design of the work, drafting the work, revising the manuscript critically for important intellectual content, final approval of the version to be published and agreement to be accountable for all aspects of the work.

Conflict of interest: The authors declared no conflict of interest.

Funding: The authors declared no funding for the study.

Conference presentation: The authors presented this research in the 45th Annual Meeting of Dermatological Society of Thailand (DST), Bangkok, Thailand in February 2020.

Ethics approval: The study was approved by the Ethics Committee of the Institute of Dermatology, Bangkok, Thailand (IRB/IEB $022 / 2562$ ) and was performed in accordance with the World Medical Association's Helsinki Declaration and its amendment.

Please cite this article as: Tiyawatanaroj $A$, Sudtikoonaseth P, Chayangsu O. Basal cell carcinoma trends in Thailand: A 10-year retrospective study of demographic, clinical and histopathological features. Dermatol Rep 2022;14:9413.

Received for publication: 1 November 2021. Accepted for publication: 27 December 2021

This work is licensed under a Creative Commons Attribution-NonCommercial 4.0 International License (CC BY-NC 4.0).

( $C$ Copyright: the Author(s), 2022

Licensee PAGEPress, Italy

Dermatology Reports 2022; 14:9413

doi:10.4081/dr.2022.9413 
py is statistically significant because of the small size of our sample.

Our non-randomized controlled trial supports the influence of a high glycemic diet on the aggravation of acne and the defensive role of the Mediterranean diet suggesting resveratrol and olive oil as protective foods. The promising implications of medical nutrition therapy in acne pathogenesis may be a viable treatment option for patients with acne, due to the diet-induced endocrine effects on acne-promoting pathways. The results of our study may highlight suitable nutrients, such as resveratrol and olive oil that can have a beneficial potential on this disease, hence we consider the Mediterranean diet useful both for Mediterranean and non-Mediterranean populations.

In any case, further clinical and translational research is necessary to investigate and confirm the association between acne and nutrition.

\section{References}

1. Karadağ AS, Balta İ, Saricaoğlu H, et al. The effect of personal, familial, and environmental characteristics on acne vulgaris: a prospective, multicenter, case controlled study. G Ital Dermatol Venereol 2019;154:177-85.

2. Spencer EH, Ferdowsian HR, Barnard ND. Diet and acne: a review of the evidence. Int J Dermatol 2009;48:339-47.

3. Melnik BC, Schmitz G. Role of insulin, insulin-like growth factor-1, hyperglycemic food and milk consumption in the pathogenesis of acne vulgaris. Exp Dermatol 2009;18:833-41.

4. Kaaks R, Bellati C, Venturelli E, et al.
Effects of dietary intervention on IGF-I and IGF-binding proteins, and related alterations in sex steroid metabolism: the Diet and Androgens (DIANA) Randomised Trial. Eur J Clin Nutr 2003;57:1079-88.

5. Tosti V, Bertozzi B, Fontana L. Health Benefits of the Mediterranean Diet: Metabolic and Molecular Mechanisms. J Gerontol A Biol Sci Med Sci 2018;73:318-26.

6. Babio N, Bulló M, Basora J, et al. Adherence to the Mediterranean diet and risk of metabolic syndrome and its components. Nutr Metab Cardiovasc Dis 2009;19:563-70.

7. Doshi A, Zaheer A, Stiller MJ. A comparison of current acne grading systems and proposal of a novel system. Int $\mathrm{J}$ Dermatol 1997;36:416-8.

8. Cordain L, Lindeberg S, Hurtado M, et al. Acne Vulgaris: A Disease of Western Civilization. Arch Dermatol 2002;138:1584-90.

9. Karadag AS, Ertugrul DT, Tutal E, Akin KO. Short-term isotretinoin treatment decreases insulin-like growth factor-1 and insulin-like growth factor binding protein-3 levels: does isotretinoin affect growth hormone physiology? $\mathrm{Br} \mathrm{J}$ Dermatol 2010;162:798-802.

10. Rodighiero E, Bertolani M, Saleri R et al. Do acne treatments affect insulinlike growth factor-1 serum levels? A clinical and laboratory study on patients with acne vulgaris. Dermatol Ther 2020; 13439

11. Tasli L, Turgut S, Kacar N, et al. Insulin-like growth factor-I gene polymorphism in acne vulgaris. J Eur Acad Dermatol Venereol 2013;27:254-7.
12. Melnik B. Dietary intervention in acne: Attenuation of increased mTORC1 signaling promoted by Western diet. Dermatoendocrinol 2012;4:20-32.

13. Jung JY, Yoon MY, Min SU, et al. The influence of dietary patterns on acne vulgaris in Koreans. Eur J Dermatol 2010;20:1-5.

14. Willett WC, Sacks F, Trichopoulou A, et al. Mediterranean diet pyramid: a cultural model for healthy eating. Am J Clin Nutr 1995;61:1402S-6S.

15. Martínez-González MÁ, Hershey MS, Zazpe I, Trichopoulou A. Transferability of the Mediterranean Diet to Non-Mediterranean Countries. What Is and What Is Not the Mediterranean Diet. Nutrients 2017;9: 1226.

16. Skroza N, Tolino E, Semyonov L, et al. Mediterranean diet and familial dysmetabolism as factors influencing the development of acne. Scand J Public Health 2012;40:466-74.

17. Claudel JP, Auffret N, Leccia MT, et al. Acne and nutrition: hypotheses, myths and facts. J Eur Acad Dermatol Venereol 2018;32:1631-7.

18. Donnarumma M, Savastano S, Barrea $\mathrm{L}$, et al. Comment on 'Acne and nutrition: hypotheses, myths and facts'. J Eur Acad Dermatol Venereol 2019;33:e418-9.

19. Barrea L, Donnarumma M, Cacciapuoti $\mathrm{S}$, et al. Phase angle and Mediterranean diet in patients with acne: Two easy tools for assessing the clinical severity of disease. J Transl Med 2021;19:171. 
tor. Karadag et al. ${ }^{9}$ in 2010 assumed and demonstrated that IGF-1 levels decreased after short-term isotretinoin treatment. Recently, Rodighiero et al. have confirmed that common therapies for acne are able to modulate the systemic route of IGF-1, but not to reduce it with statistical significance. ${ }^{10}$

Circulating levels of IGF-1 are used to evaluate the bioactivity of IGF-1 on tissue because of the lack of alternative techniques in vivo. Its bioactivity depends not only on IGF-1 serum levels but also on IGF binding protein levels, on genetic factors and on local production. Even though different elements such as diet, age, sex or hormones can influence IGF-1 levels, more than $60 \%$ is genetically determined. Hence it has been suggested by Tasli et al. that the only way to adjust IGF-1 levels is to act on the unique manageable factor, represented by diet. ${ }^{11}$ Furthermore, several previous randomized controlled trials found that a MD may reduce the biological activity of IGF-1. ${ }^{5}$

According to Melnik, dietary intervention in acne patients should decrease fat, glucose and total energy intake; furthermore, the diet can diminish insulin/IGF-1 signaling, which is mainly mediated by milk protein assumption. The same author proposes an increase in fruit and vegetable consumption, reducing animal derived food and recommends a paleolithic diet. ${ }^{12}$

Interestingly, Jung et al have shown a lower incidence of acne in Koreans adhering to a traditional Korean diet, with a low glycemic index and low-fat content and, conversely, a higher incidence in people following a westernized diet. ${ }^{13}$

Along with these factors we have hypothesized that the MD could be the most suitable diet for our patients. It is characterized by abundant plant foods (fruit, vegetables, bread, other forms of cereals, potatoes, beans, nuts, and seeds), fresh fruit as the typical daily dessert, low to moderate consumption of fish, poultry, eggs and dairy products (predominantly cheese and yogurt) and low amounts of red meat. ${ }^{14}$ The main sources of fat and alcohol are primarily virgin olive oil and wine respectively. They contain hydroxytyrosol and tyrosol, oleocanthal, resveratrol, and many other dietary bioactive phenolic compounds with substantial anti-inflammatory properties. Consequently, the Mediterranean diet is rich in antioxidant compounds and bioactive elements with anti-inflammatory effects and has a low glycemic index. ${ }^{15}$ It is well known that this dietary pattern is healthy and has positive effects on longevity, quality of life and disease prevention such as metabolic syndrome, cardiovascular diseases and could reduce the incidence of cancers. ${ }^{14}$ Concerning acne pathogenesis, the protective role of Mediterranean diet was first assumed and demonstrated by Skroza et al. ${ }^{16}$ and, more recently, confirmed by the studies of Claudel et al. ${ }^{17}$ Latterly, Donnarumma et al. ${ }^{18}$ suggested a possible protective role of resveratrol on acne by investigating adherence to the Mediterranean diet, using the PREDIMED questionnaire, and the severity of acne in patients and controls. Barrea et al. ${ }^{19}$ affirmed that high adherence to the MD could be a marker of inflammation in patients with acne and the PREDIMED score $\leq 9$ predicted the highest GAGS score. They suggested that the body composition and PREDIMED scores might represent possible markers of the severity of acne in a clinical setting.

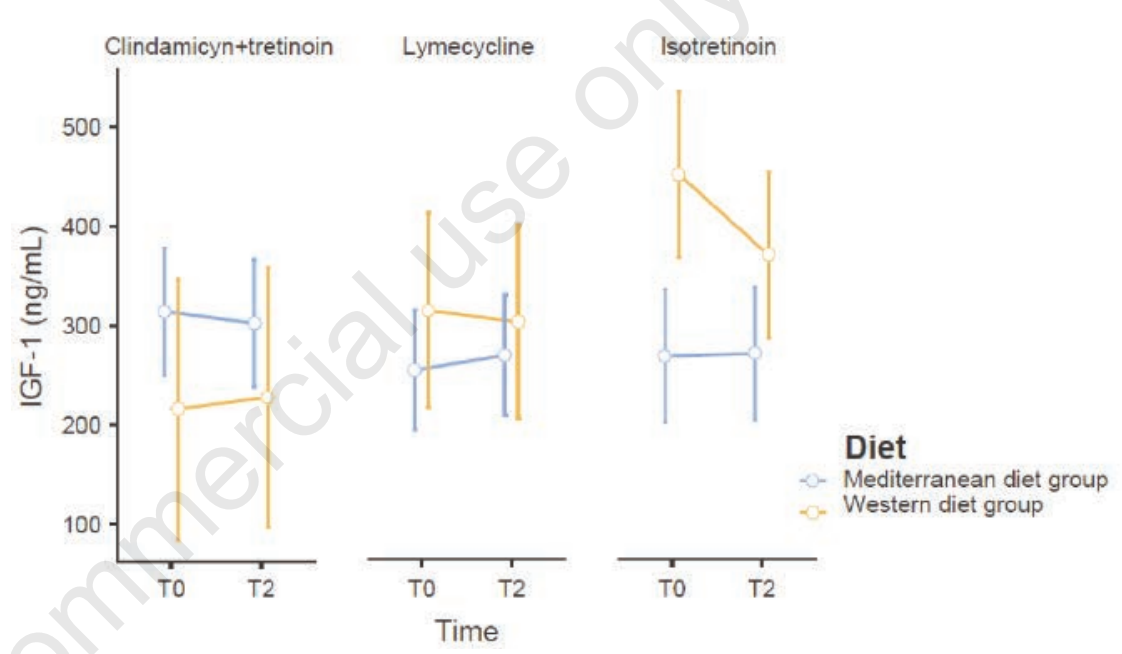

Figure 2. Mean values and 95\% confidence interval (95\%-CI) of IGF-1 levels stratified by the type of treatment and diet at T0 (first visit) and T2 (end of acne treatment).
In light of this evidence, we have investigated not only adherence to the Mediterranean diet of patients affected by acne vulgaris, but also considered the possible relationship with IGF-1 levels. In our experience, patients with a high-average adherence to the MD exhibited lower IGF-1 serum levels compared to patients that did not follow the MD and this difference is statistically significant (Figure 1). Intriguingly, we found that IGF-1 serum levels of patients with severe acne and low adherence to the Mediterranean diet are reduced after six months of systemic isotretinoin intake (Figure 2), confirming the fact that this therapy is able to modulate the levels of IGF-1 only in patients that do not follow the MD. ${ }^{8}$ Nevertheless we cannot state that this reduction of IGF-1 serum levels after thera-

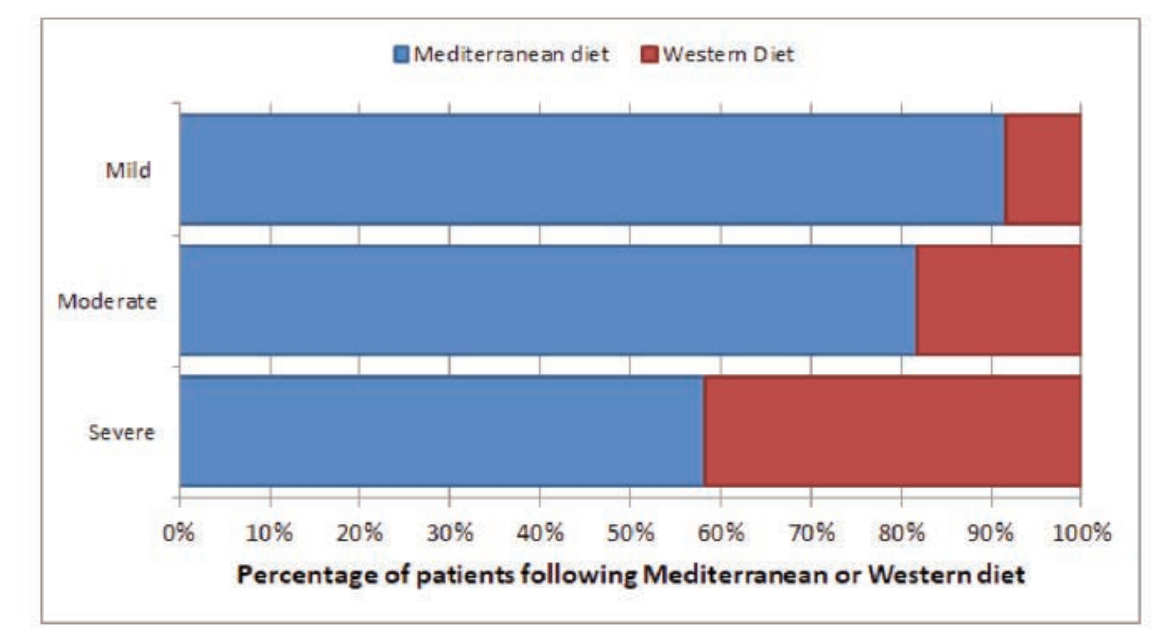

Figure 3. Percentage of patients following Mediterranean diet or Western diet divided according to acne severity.

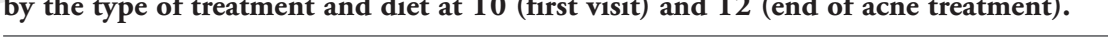


tionnaire was considered valid only when all 14 items were completed. Participants who scored $\geq 6$ points were considered compliant with the MD and were defined Mediterranean Diet Group (MD-Group), who scored $<5$ points were considered compliant with Western Diet and formed the Western Diet Group (WD-Group). The control group was composed of 15 agematched subjects without clinically manifest acne or its consequences at the time of the study.

The subjects' age, gender, body mass index (BMI), waist circumference and severity of acne were recorded. Patients with endocrinological pathologies, those who were underweight, overweight, or subjects with other diseases or on medication that could interfere with IGF-1 were excluded from the study.

A clinical diagnosis of acne vulgaris was made after examining the patients in a proper daylight-illuminated examination room by an experienced dermatologist. Scoring of the severity of acne vulgaris was evaluated by the same dermatologist with the Global Acne Grading System (GAGS). ${ }^{7}$

The GAGS score range of our patients was between 1 and 38 , so we considered three groups. Every group received a treatment chosen according to the recent clinical European Dermatology Forum guidelines and to acne severity. Specifically, patients with mild acne (GAGS $\leq 18$ ) received Clindamycin $10 \mathrm{mg} / \mathrm{g}+$ tretinoin $0.25 \mathrm{~g}$ gel for 3 months; the ones with moderate acne (GAGS 19-30) received oral lymecycline $300 \mathrm{mg}+$ topical benzoyl peroxide $5 \%$ gel for 3 months; the ones with severe acne (GAGS $\geq 31$ ) received Isotretinoin $0,5 \mathrm{mg} / \mathrm{kg} /$ die for 8 months (total cumulative dose of $120 \mathrm{mg} / \mathrm{kg}$ ). The clinical evaluation relies on 3 medical examinations: T0 (first visit), T1 (1 month treatment) and T2 (end of treatment). For patients with severe acne the follow up was every 2 months for 8 months. During each examination, the GAGS score has been calculated, possible side-effects has been investigated and any change in patients' life quality documented.

Blood was drawn from each fasting participant during T0 and during T2 (after 3 months for patients with mild and moderate acne and after 8 months for patients with severe acne). Blood samples were drawn by venipuncture and centrifuged by a trained phlebotomist; the serum was separated and stored at $-25^{\circ} \mathrm{C}$. In order to avoid variation, all samples were studied on the same day and using the same kit. IGF-1 levels were determined by an IDS-iSYS automated IGF-I immunoassay (IDS-iSYS Insulin-like Growth Factor-I, Immunodiagnostic Systems Limited, Boldon, Tyne \& Wear,
NE35 9PD, UK). The study was conducted according to the rules of good clinical practice and the study protocol was approved by the local ethics committee. Eligible participants were recruited at the Dermatology Clinic of Parma between Dec 2018 and Dec 2019. Before enrollment, written consent was obtained from each participant or guardian (if the participant was aged $<18$ years).

\section{Results}

We analyzed 35 patients: 25 female and 10 males with a mean age of $21.02 \pm 4.8$ years; 27 patients belong to the MD group and 8 belong to the WD Group. Data were compared to a control group including 7 males and 6 female subjects, with a mean age of $26.60 \pm 2.16$ years. Serum IGF-1 levels were within the normal reference range. There is a statistically significant difference $(\mathrm{P}=0.05)$ in serum IGF-1 levels between controls and patients, with higher values in the patient group.

At $\mathrm{T} 0$ the medium value of IGF-1 serum levels of the MD group was 270,9 $\mathrm{ng} / \mathrm{mL}$ and for the WD group was 350,5 $\mathrm{ng} / \mathrm{mL}$ : the difference between the two groups is statistically significant $(\mathrm{P}=0.027)$.

There is a statistically significant decrease of IGF-1 level for the patients belonging to the WD group after two months of acne therapy. Conversely, the patients of the MD group have a lower serum IGF-1 value at T0 with no variation after two months of acne therapy (Figure 1)

Similar results were obtained when we considered only the patients with severe acne, who were treated with isotretinoin. Specifically, there is a statistically significant difference in IGF-1 serum levels between the WD group and the MD group at T0. In addition, for the first group there is a reduction of IGF-1 serum levels after six months of therapy.

In patients with mild and moderate acne, there is no statistically significant reduction of IGF-1 serum levels after topical or systemic antibiotic treatment in both groups (Figure 2)

Acne severity at baseline differed between MD and WD patients even if there is not a statistically significant difference due to the small sample size (Figure 3).

\section{Discussion and conclusions}

Although the past dermatological doctrine denied a direct involvement of diet in acne pathogenesis, the most recent research has shown a relationship between excessive intake of dairy products, hyperglycaemic food and acne. ${ }^{8}$ Current knowledge supports the evidence that a Western diet induces hyperinsulinemia and subsequently raises IGF-1 serum levels which may play a crucial role in the pathophysiology of acne. In fact, IGF-1 stimulates sebocyte growth, lipogenesis of sebaceous glands, androgen synthesis and promotes proliferation of keratinocytes via activation of the IGF-1 recep-

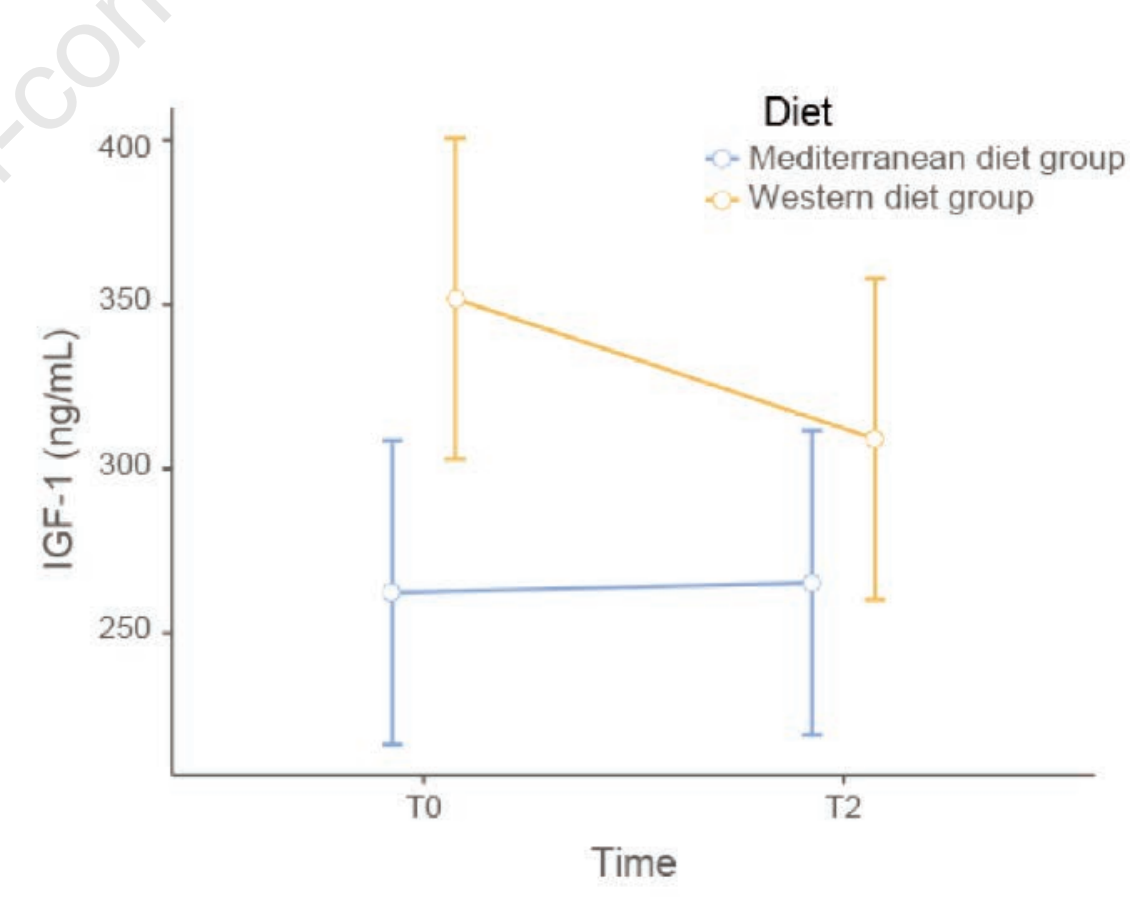

Figure 1. Mean values and 95\% confidence interval (95\%-CI) of IGF-1 levels stratified by the type of diet at T0 (first visit) and T2 (end of acne treatment). 


\section{The influence of Mediterranean diet in acne pathogenesis and the correlation with insulin-like growth factor-1 serum levels: Implications and results}

\author{
Mariabeatrice Bertolani, ${ }^{1}$ \\ Eleonora Rodighiero, ${ }^{1}$ Roberta Saleri, ${ }^{2}$ \\ Giuseppe Pedrazzi, ${ }^{3}$ Simona Bertoli, ${ }^{4,5}$ \\ Alessandro Leone, ${ }^{4}$ Claudio Feliciani, ${ }^{1}$ \\ Torello Lotti, ${ }^{6,7}$ Francesca Satolli ${ }^{1,8}$
}

${ }^{1}$ Section of Dermatology, Department of Medicine and Surgery, University of Parma; ${ }^{2}$ Department of Veterinary Science, University of Parma; ${ }^{3}$ Department of Medicine and Surgery, Unit of Neuroscience and Interdepartmental Centre of Robust Statistics, University of Parma;

${ }^{4}$ International Center for the Assessment of Nutritional Status, Department of Food, Environmental and Nutritional Sciences (DeFENS), University of Milan; 5Istituto Auxologico Italiano, IRCCS, Lab of Nutrition and Obesity Research, Milan; 'University Unit of Dermatology and Regenerative Medicine, University of Rome G. Marconi, Italy; ${ }^{7}$ Department of Dermatology and Communicable Diseases, Ministry of Health, Moscow Russia First Medical Moscow State University, Higher Education Institution I. M. Sechenov, Russian Federation; ${ }^{8}$ Dermatologic Unit, University Hospital of Parma, Italy

\footnotetext{
Abstract

Acne is a chronic inflammatory disease of the pilosebaceous unit, and its etiology is complex and multifactorial. The role of the diet in its pathogenesis is still debated. The purpose of this study was to assess the association between MD and IGF-1 in acne patients and, as secondary objective, the role of systemic treatment on IGF-1 serum levels, in accordance with the patients' diet. This study included 35 patients aged 14-30 years affected by acne and treated in line with the EDF guidelines. Patients were divided into 2 groups based on a questionnaire score assessing the adherence to the Mediterranean diet: the Mediterranean Group (score $\geq 6$ ) and the Western Group $($ score $<5)$. IGF-1 serum levels were measured in all patients before and after treatment and then compared to healthy population. IGF-1 levels were higher in patients
}

than in controls and in the Western group than in the Mediterranean group. We speculate that the Mediterranean diet can have a protective role in the pathogenesis of acne by acting on the systemic route of IGF-1.

\section{Introduction}

Acne vulgaris is the most common chronic inflammatory disease of the pilosebaceus unit. It affects almost all young adults aged 15-17; although its prevalence tends to decrease with age, $15-20 \%$ of adults are affected resulting in negative effects on their self-esteem, mood and quality of life. ${ }^{1}$ Its etiology appears to be complex and multifactorial, involving abnormal follicular keratinization, hyperseborrhoea, Cutibacterium Acnes proliferation in the pilosebaceous unit and inflammatory mediators released into the skin. The role of diet in the literature remains controversial but recent studies have supported the relationship between specific dietary factors and acne. ${ }^{2}$ In particular, an association with dairy products and with high-glycaemicindex foods has been demonstrated. ${ }^{3}$

Diet based on products with a high glycemic index leads to hyperinsulinemia affecting the level of circulating Insulin Like Growth Factor-1 (IGF-1), which has been proposed to have a key role in acne pathogenesis and acne has been proposed to be an IGF-1-mediated disease. ${ }^{3}$ The regulation of IGF-1 by diet is complex, however, several previous randomized controlled trials found that a Mediterranean diet (MD) may reduce the biological activity of IGF1.,5 The typical MD pattern is characterized by low-glycaemic-index foods, low amount of dairy products, high amount of antioxidant and antinflammatory nutrients, that may be able to modulate IGF-1 plasma concentration. Moreover, given the existing evidence on acne and inflammatory pathways, the antioxidant and anti-inflammatory effects of MD may be protective against the onset of diseases. However, no previous observational or prospective cohort study has been conducted to assess the role of MD on acne in relation to IGF-1 plasma concentration modulation and on the effect of drugs treatment on IGF-1 plasma concentration according to baseline dietary pattern. ${ }^{6}$ The purpose of this study was to assess the association between MD and IGF-1 in acne patients and, as secondary objective, the role of systemic treatment on IGF-1 serum levels, in accordance with the patients' diet.
Correspondence: Eleonora Rodighiero, Dermatology Unit, Department of Medicine and Surgery, University of Parma, Via Gramsci 14, 43126 Parma, Italy.

Tel.: +39-0521-033562 - Fax: +39-0521702959.

E-mail: eleonora.rodighiero@gmail.com

Key words: Acne; Insulin Like Growth Factor-1; IGF-1; Mediterranean diet.

Acknowledgements: The authors would like to thank Rosalia Aloe.

Contributions: MB: conceived and designed the analysis, collected the data and wrote the paper. ER: conceived and designed the analysis, collected the data and wrote the paper. RS: contributed data and analysis tools. GP: performed the analysis. SB: contribuited to the analysis and interpretation of data of the work. AL: contributed to the analysis and interpretation of data of the work. CF: conceived and designed the analysis. TL: conceived and designed the analysis. FS: conceived and designed the analysis, collected the data and wrote the paper.

Conflict of interest: The authors declare that they have no conflict of interest.

Funding: None.

Availability of data and material: Data and materials are available by the authors.

Informed consent:??

Please cite this article as: Bertolani $M$, Rodighiero E, Saleri R, et al. The influence of Mediterranean diet in acne pathogenesis and the correlation with insulin-like growth factor1 serum levels: implications and results. Dermatol Rep 2022;14:9143.

Received for publication: 18 March 2021. Accepted for publication: 29 November 2021.

This work is licensed under a Creative Commons Attribution-NonCommercial 4.0 International License (CC BY-NC 4.0).

OCopyright: the Author(s), 2022

Licensee PAGEPress, Italy

Dermatology Reports 2022; 14:9143

doi:10.4081/dr.2022.9143

\section{Materials and methods}

This study included 35 participants with acne vulgaris aged 14-30 years who were affected by mild, moderate, and severe acne. Adherence to the traditional MD was assessed using a validated 14-item questionnaire ${ }^{6}$. Values of 0 or 1 were assigned to each of 14 dietary components. The ques- 
Family Med Prim Care 2019;8:3496503.

75. Langan EA, Griffiths CEM, Solbach W, et al. The role of the microbiome in psoriasis: moving from disease description to treatment selection? Br J Dermatol 2018;178:1020-7.

76. Chimenti MS, Perricone C, Novelli L, et al. Interaction between microbiome and host genetics in psoriatic arthritis. Autoimmun Rev 2018;17:276-83.

77. Eppinga H, Konstantinov SR, Peppelenbosch MP, Thio HB. The microbiome and psoriatic arthritis. Curr
Rheumatol Rep 2014;16:407.

78. Chen YJ, Ho HJ, Tseng $\mathrm{CH}$, et al. Intestinal microbiota profiling and predicted metabolic dysregulation in psoriasis patients. Exp Dermatol 2018;27:1336-43.

79. Smith PM, Howitt MR, Panikov N, et al. The microbial metabolites, shortchain fatty acids, regulate colonic Treg cell homeostasis. Science 2013;341:569-73.

80. Fine LM, Bernstein JA. Guideline of Chronic Urticaria Beyond. Allergy Asthma Immunol Res 2016;8:396-403.
81. Kayiran MA, Akdeniz N. Diagnosis and treatment of urticaria in primary care. North Clin Istanb. 2019;6:93-9.

82. Zuberbier T, Aberer W, Asero R, et al. The EAACI/GA(2) LEN/EDF/WAO Guideline for the definition, classification, diagnosis, and management of urticaria: the 2013 revision and update. Allergy 2014;69:868-87.

83. Polkowska-Pruszyńska B, Gerkowicz A, Krasowska D. The gut microbiome alterations in allergic and inflammatory skin diseases - an update. J Eur Acad Dermatol Venereol 2020;34:455-64. 
63.

35. Reddel S, Del Chierico F, Quagliariello A, et al. Gut microbiota profile in children affected by atopic dermatitis and evaluation of intestinal persistence of a probiotic mixture. Sci Rep 2019;9:4996.

36. Ye S, Yan F, Wang H, et al. Diversity analysis of gut microbiota between healthy controls and those with atopic dermatitis in a Chinese population. $\mathrm{J}$ Dermatol 2020.

37. Adlerberth I, Strachan DP, Matricardi PM, et al. Gut microbiota and development of atopic eczema in 3 European birth cohorts. J Allergy Clin Immunol 2007;120:343-50.

38. Kirjavainen PV, Arvola T, Salminen SJ, Isolauri E. Aberrant composition of gut microbiota of allergic infants: a target of bifidobacterial therapy at weaning? Gut 2002;51:51-5.

39. Codoñer FM, Ramírez-Bosca A, Climent E, et al. Gut microbial composition in patients with psoriasis. Sci Rep 2018;8:3812.

40. Hidalgo-Cantabrana C, Gómez J, Delgado $\mathrm{S}$, et al. Gut microbiota dysbiosis in a cohort of patients with psoriasis. Br J Dermatol 2019;181:1287-95.

41. Huang L, Gao R, Yu N, et al. Dysbiosis of gut microbiota was closely associated with psoriasis. Sci China Life Sci 2019;62:807-15.

42. Scher JU, Ubeda C, Artacho A, et al. Decreased bacterial diversity characterizes the altered gut microbiota in patients with psoriatic arthritis, resembling dysbiosis in inflammatory bowel disease. Arthritis Rheumatol 2015;67:128-39.

43. Shapiro J, Cohen NA, Shalev V, et al. Psoriatic patients have a distinct structural and functional fecal microbiota compared with controls. J Dermatol 2019;46:595-603.

44. Eppinga H, Sperna Weiland CJ, Thio HB, et al. Similar Depletion of Protective Faecalibacterium prausnitzii in Psoriasis and Inflammatory Bowel Disease, but not in Hidradenitis Suppurativa. J Crohns Colitis 2016;10:1067-75.

45. Dei-Cas I, Giliberto F, Luce L, et al. Metagenomic analysis of gut microbiota in non-treated plaque psoriasis patients stratified by disease severity: development of a new PsoriasisMicrobiome Index. Sci Rep 2020;10:111.

46. Tan L, Zhao S, Zhu W, et al. The Akkermansia muciniphila is a gut microbiota signature in psoriasis. Exp Dermatol 2018;27:144-9.
47. Yegorov S, Babenko D, Kozhakhmetov $\mathrm{S}$, et al. Psoriasis Is Associated With Elevated Gut IL-1 $\alpha$ and Intestinal Microbiome Alterations. Front Immunol 2020;11:571319.

48. Lu T, Chen Y, Guo Y, et al. Altered Gut Microbiota Diversity and Composition in Chronic Urticaria. Dis Markers 2019;2019:6417471.

49. Nabizadeh E, Jazani NH, Bagheri M, Shahabi S. Association of altered gut microbiota composition with chronic urticaria. Ann Allergy Asthma Immunol 2017;119:48-53.

50. Rezazadeh A, Shahabi S, Bagheri M, et al. The protective effect of Lactobacillus and Bifidobacterium as the gut microbiota members against chronic urticaria. Int Immunopharmacol 2018;59:168-73.

51. Wang D, Guo S, He H, et al. Gut Microbiome and Serum Metabolome Analyses Identify Unsaturated Fatty Acids and Butanoate Metabolism Induced by Gut Microbiota in Patients With Chronic Spontaneous Urticaria. Front Cell Infect Microbiol 2020;10:24.

52. Thursby E, Juge N. Introduction to the human gut microbiota. Biochem $\mathrm{J}$ 2017;474:1823-36.

53. Zhang H, Liao W, Chao W, et al. Risk factors for sebaceous gland diseases and their relationship to gastrointestinal dysfunction in Han adolescents. J Dermatol 2008;35:555-61.

54. Eichenfield LF, Del Rosso JQ, Mancini AJ, et al. Evolving perspectives on the etiology and pathogenesis of acne vulgaris. J Drugs Dermatol 2015;14:26372.

55. Dreno B. What is new in the pathophysiology of acne, an overview. J Eur Acad Dermatol Venereol 2017;31:8-12.

56. Bowe W, Patel NB, Logan AC. Acne vulgaris, probiotics and the gut-brainskin axis: from anecdote to translational medicine. Benef Microbes 2014;5:18599.

57. Bowe WP, Logan AC. Acne vulgaris, probiotics and the gut-brain-skin axis back to the future? Gut Pathog 2011;3:1.

58. Gupta VK, Paul S, Dutta C. Geography, Ethnicity or Subsistence-Specific Variations in Human Microbiome Composition and Diversity. Front Microbiol 2017;8:1162.

59. Maarouf M, Platto JF, Shi VY. The role of nutrition in inflammatory pilosebaceous disorders: Implication of the skingut axis. Australas J Dermatol 2019;60:e90-e8.

60. Shin N-R, Whon TW, Bae J-W. Proteobacteria: microbial signature of dysbiosis in gut microbiota. Trends Biotechnol 2015;33:496-503.

61. Kwon HK, Lee CG, So JS, et al. Generation of regulatory dendritic cells and CD4+Foxp3 $+\mathrm{T}$ cells by probiotics administration suppresses immune disorders. Proc Natl Acad Sci USA 2010;107:2159-64.

62. Madsen K, Cornish A, Soper P, et al. Probiotic bacteria enhance murine and human intestinal epithelial barrier function. Gastroenterology 2001;121:58091.

63. Resta-Lenert S, Barrett KE. Probiotics and commensals reverse TNF-alphaand IFN-gamma-induced dysfunction in human intestinal epithelial cells. Gastroenterology 2006;130:731-46.

64. Dinulos JG, Trickett A, Crudele C. New science and treatment paradigms for atopic dermatitis. Curr Opin Pediatr 2018;30:161-8.

65. Thomas CL, Fernández-Peñas P. The microbiome and atopic eczema: More than skin deep. Australas J Dermatol 2017;58:18-24.

66. Brüssow H. Turning the inside out: the microbiology of atopic dermatitis. Environ Microbiol 2016;18:2089-102.

67. Sjogren YM, Jenmalm MC, Bottcher MF, Bjorksten B, Sverremark-Ekstrom E. Altered early infant gut microbiota in children developing allergy up to 5 years of age. Clin Exp Allergy 2009;39:518-26.

68. Zákostelská Z, Málková J, Klimešová $\mathrm{K}$, et al. Intestinal Microbiota Promotes Psoriasis-Like Skin Inflammation by Enhancing Th17 Response. PLoS One 2016;11:e0159539.

69. Komine M. Recent Advances in Psoriasis Research; the Clue to Mysterious Relation to Gut Microbiome. Int J Mol Sci 2020;21.

70. Jinrong Zeng SL, Yumeng Huang, Qianjin Lu. Critical role of environmental factors in the pathogenesis of psoriasis. J Dermatol 2017:10.

71. Rendon A, Schakel K. Psoriasis Pathogenesis and Treatment. Int $\mathrm{J}$ Mol Sci 2019;20.

72. Zeng J, Luo S, Huang Y, Lu Q. Critical role of environmental factors in the pathogenesis of psoriasis. J Dermatol 2017;44:863-72.

73. Algazina T, Yermekbayeva B, Batpenova G, Kushugulova A. Features of microbiota in psoriatic disease: from skin and gut perspectives (review) Georgian Med News 2019:98-104.

74. Alesa DI, Alshamrani HM, Alzahrani YA, et al. The role of gut microbiome in the pathogenesis of psoriasis and the therapeutic effects of probiotics. J 
leptum, genus Lactobacillus, and Bifidobacterium have shown significant prevention of other allergic and inflammatory diseases. ${ }^{83}$

The mechanisms of protective effects of those anti-inflammatory bacteria against CU can be due to the induction of regulatory $\mathrm{T}$ (Treg) cells by these bacteria. The intestinal microbiota composition could lower the $\mathrm{pH}$ level, modulate the integrity of the epithelial cell barrier, and regulate mucus secretion, which altered the function and expression of tight junction protein. ${ }^{49,50}$ The further studies are needed to reveal the pathway of this observation.

\section{Conclusions}

The gut microbiome in inflammatory skin disorders such as atopic dermatitis, acne vulgaris, urticaria, and psoriasis tend to be altered and imbalanced. However, the role of specific microbiota as the marker of skin inflammation still needs further study. The Bifidobacterium tends to decrease in all skin disorder reviewed in these articles and increases in E. coli and Proteobacteria are shown in inflammatory skin disorders. The role of Bacteroidetes and Firmicutes is still conflicting and inconsistent in many articles.

\section{References}

1. Dethlefsen L, McFall-Ngai M, Relman DA. An ecological and evolutionary perspective on human-microbe mutualism and disease. Nature 2007;449:8118.

2. Murillo N, Raoult D. Skin microbiota: overview and role in the skin diseases acne vulgaris and rosacea. Future Microbiol 2013;8:209-22.

3. Knight R, Callewaert C, Marotz C, et al. The Microbiome and Human Biology. Annu Rev Genomics Hum Genet 2017; 18:65-86.

4. Schlaeppi K, Bulgarelli D. The plant microbiome at work. Mol Plant Microbe Interact 2015;28:212-7.

5. Gill T, Brooks SR, Rosenbaum JT, et al. Novel Inter-omic Analysis Reveals Relationships Between Diverse Gut Microbiota and Host Immune Dysregulation in HLA-B27-Induced Experimental Spondyloarthritis. Arthritis Rheumatol 2019;71:1849-57.

6. Petrosino JF, Highlander S, Luna RA, et al. Metagenomic pyrosequencing and microbial identification. Clin Chem 2009;55:856-66.

7. van der Meulen TA, Harmsen $\mathrm{H}$,
Bootsma H, et al. The microbiome-systemic diseases connection. Oral Dis 2016;22:719-34.

8. Qin Y, Wade PA. Crosstalk between the microbiome and epigenome: messages from bugs. J Biochem 2018;163:10512.

9. Structure, function and diversity of the healthy human microbiome. Nature 2012;486:207-14.

10. Grice EA, Segre JA. The human microbiome: our second genome. Annu Rev Genomics Hum Genet 2012;13:151-70.

11. Gevers D, Knight R, Petrosino JF, et al. The Human Microbiome Project: a community resource for the healthy human microbiome. PLoS Biol 2012; 10:e1001377.

12. Cresci GA, Bawden E. Gut Microbiome: What We Do and Don't Know. Nutr Clin Pract 2015;30:734-46.

13. Mowat AM, Agace WW. Regional specialization within the intestinal immune system. Nat Rev Immunol 2014;14:667-85.

14. Cani PD. Human gut microbiome: hopes, threats and promises. Gut 2018;67:1716-25.

15. Tralau T, Sowada J, Luch A. Insights on the human microbiome and its xenobiotic metabolism: what is known about its effects on human physiology? Expert Opin Drug Metab Toxicol 2015;11:41125.

16. Actis GC. The gut microbiome. Inflamm Allergy Drug Targets 2014;13:217-23.

17. Arck P, Handjiski B, Hagen E, et al. Is there a 'gut-brain-skin axis'? Exp Dermatol 2010;19:401-5.

18. Moher D, Liberati A, Tetzlaff J, et al. Preferred reporting items for systematic reviews and meta-analyses: the PRISMA statement. PLoS Med 2009;6:e1000097.

19. Deng Y, Wang H, Zhou J, et al. Patients with Acne Vulgaris Have a Distinct Gut Microbiota in Comparison with Healthy Controls. Acta Derm Venereol 2018;98:783-90.

20. Yan HM, Zhao HJ, Guo DY, et al. Gut microbiota alterations in moderate to severe acne vulgaris patients. J Dermatol 2018;45:1166-71.

21. Thompson KG, Rainer BM, Antonescu $\mathrm{C}$, et al. Minocycline and Its Impact on Microbial Dysbiosis in the Skin and Gastrointestinal Tract of Acne Patients. Ann Dermatol 2020;32.

22. Rainer BM, Thompson KG, Antonescu $\mathrm{C}$, et al. Impact of lifestyle and demographics on the gut microbiota of acne patients and the response to minocycline. J Dermatolog Treat 2020:1-2.
23. Kingkaw A, Nakphaichit $M$, Suratannon N, et al. Analysis of the infant gut microbiome reveals metabolic functional roles associated with healthy infants and infants with atopic dermatitis using metaproteomics. Peer J 2020;8:e9988.

24. Lee E, Lee SY, Kang MJ, et al. Clostridia in the gut and onset of atopic dermatitis via eosinophilic inflammation. Ann Allergy Asthma Immunol 2016;117:91-2.e1

25. Lee MJ, Kang MJ, Lee SY, et al. Perturbations of gut microbiome genes in infants with atopic dermatitis according to feeding type. J Allergy Clin Immunol 2018;141:1310-9.

26. Mahdavinia M, Rasmussen HE, Botha M, et al. Effects of diet on the childhood gut microbiome and its implications for atopic dermatitis. J Allergy Clin Immunol 2019;143:1636-7.e5.

27. Mahdavinia M, Rasmussen HE, Engen $\mathrm{P}$, et al. Atopic dermatitis and food sensitization in South African toddlers: Role of fiber and gut microbiota. Ann Allergy Asthma Immunol 2017;118:742-3.e3.

28. Oh S, Yap GC, Hong P-Y, et al. Immune-modulatory genomic properties differentiate gut microbiota of infants with and without eczema. PLoS ONE 2017;12:1-16.

29. Park YM, Lee SY, Kang MJ, et al. Imbalance of Gut Streptococcus, Clostridium, and Akkermansia Determines the Natural Course of Atopic Dermatitis in Infant. Allergy Asthma Immunol Res 2020;12:322-37.

30. Penders J, Stobberingh EE, Thijs C, et al. Molecular fingerprinting of the intestinal microbiota of infants in whom atopic eczema was or was not developing. Clin Exp Allergy 2006;36:1602-8.

31. Song H, Yoo Y, Hwang J, et al. Faecalibacterium prausnitzii subspecies-level dysbiosis in the human gut microbiome underlying atopic dermatitis. J Allergy Clin Immunol 2016;137:852-60.

32. Zheng $H$, Liang $H$, Wang $Y$, et al. Altered Gut Microbiota Composition Associated with Eczema in Infants. PLoS ONE 2016;11:1-13.

33. Chan CWH, Yuet Wa Chan J, et al. Altered Gut Microbiome and Environmental Factors Associated with Development of Eczema in Hong Kong Infants: A 4-Month Pilot Study. Int J Environ Res Public Health 2020;17.

34. Mah KW, Björkstén B, Lee BW, et al. Distinct pattern of commensal gut microbiota in toddlers with eczema. Int Arch Allergy Immunol 2006;140:157- 


\section{fragilis in $\mathrm{AD}$ infants. ${ }^{32}$}

Bifidobacterium spp. are assumed to be beneficial for human health due to their effects on vitamin production, immune system stimulation, inhibition of potentially pathogen bacteria, and improvement of food ingredients digestion. Eight articles reported the reduction of Bifidobacterium in AD patients compared to control. This absence of Bifidobacterium in AD children could lead to a lack of anti-inflammatory effect. ${ }^{35}$

An increased abundance of $E$. coli was reported in two articles. However, studies on the role of E. coli in the intestine are lacking. One of the plausible hypotheses is that LPS originating from $E$. coli might play a role in regulating the immune system through the intestinal epithelial cells. Lee et al. in 2018 found a positive association between the relative abundance of $E$. coli and the percentage of blood eosinophils that play a critical role in developing $\mathrm{AD}$ and regulating immune homeostasis microbiota profiles in the intestine. ${ }^{25}$

\section{Psoriasis}

Psoriasis is a chronic inflammatory disease characterized by thickening of the skin, especially in the pressure site. ${ }^{68}$ Psoriasis is also considered a systemic disease, where the cardiovascular system, insulin homeostasis, psychomotor systems, and lipid metabolism are convoluted. ${ }^{69}$ This disease affects approximately $2-4 \%$ of the world's population and is influenced by genetics, disorder of the immune system, epigenetic, and environmental factors. ${ }^{43,70}$

The pathogenesis of psoriasis involves sustained inflammation that leads to uncontrolled keratinocyte proliferation and dysfunctional differentiation. ${ }^{71} \mathrm{~T}$ cells are crucial in the pathogenesis of psoriasis, especially the critical role of Th17 cells and, recently, the role of gut and skin microbiome mediator in psoriasis. Naïve T cells are activated by antigen-presenting cells (APC) in the epidermis, which promotes naïve $\mathrm{T}$ cells to differentiate into $\mathrm{T}$-helper (Th)1 and Th17, then Th1 and Th17 cell increase while $\mathrm{Th} 2$ and regulatory $\mathrm{T}$ cells (Treg) decrease. The activated $\mathrm{T}$ cells migrate from lymph nodes to the skin, where they are stimulated to produce numerous cytokines. These cytokines interact with the resident epidermal and dermal cells and cause changes, including keratinocyte proliferation and epidermal thickness. Environmental factors appear to induce inflammatory diseases in individuals with latent genetic susceptibility. Diet, microbial infections (virus, bacteria, fungi), chemical irritants or ultraviolet (UV) radiation exposure, and bad habits (drinking and smoking) are environmental factors that could induce inflammation. The interplay between genetic and ecological factors contributes to the onset, development, and present clinical symptoms of psoriasis..$^{72}$

The gut-skin axis microbiome has been postulated to be involved in the altered immune response in psoriasis. ${ }^{69,73-77}$ Even though the finding of microbiota associated with psoriasis is conflicting, the studies revealed interesting and important changes in the gut microbiome led to the pathogenesis in psoriasis.

Firmicutes were observed as the most abundant microbiota in psoriasis and psoriasis arthritis and increased as seen in four studies. ${ }^{40,41,43,78}$ The result of a high level of Firmicutes is the disturbance the ratio of Firmicutes and Bacteroidetes. This imbalance then influences carbohydrate metabolism and alters the production of medium- and short-chain fatty acids (SCFA), resulting in increased acetate and reduced butyrate production, and finally leads to chronic inflammation and compromises the gut epithelial barrier. The lack of any gut epithelial barrier alters immune responses, both locally and systemically. ${ }^{43}$

Bacteroides produce SCFA such as propionates and butyrates, which are important in regulating colonic $\mathrm{T}$ cell regulators (Tregs) directly. Thus, the SCFA regulate intestinal homeostasis and control inflammation by limiting the proliferation of effector CD4+ T cells (Teff) ${ }^{79}$ There were conflicting data about the role of Bacteroides in psoriasis. Reduction of Bacteroidetes in psoriasis reported in other studies suggest lowering the SCFA. ${ }^{39,40,42,43,45}$ SCFA are the major metabolic products of anaerobic fermentation of glycans, where Bacteroides bind to complex recalcitrant glycan and stimulate the production of SCFA. ${ }^{45}$ The articles that demonstrated the high abundance of Bacteroides assumed the role of Bacteroides as a potential pathogen that promotes the pathogenesis of psoriasis.

Faecalibacterium prausnitzii is suggested to be causally related to the induction of psoriasis. Studies showed a reduction of $F$. prausnitzii linked to systemic inflammation in both skin and intestines due to the ability of $F$. prausnitzii on secreting anti-inflammatory molecules that modulate the host immune system. ${ }^{40} \mathrm{~F}$. prausnitzii can regulate $\mathrm{Th} 17 /$ regulatory $\mathrm{T}$ cell differentiation and reported consistently as butyrate producers in the intestine. ${ }^{45}$ However, other studies reported a high abundance of Faecalibacterium in psoriasis. ${ }^{39,43,45,47}$ This dynamic role of Faecalibacterium spp. supported the evidence of these bacteria's pathogenesis in psoriasis. The conflicting data are possibly because of different inclusion and exclusion criteria on recruiting psoriasis patients.

Akkermansia was identified as a mucolytic mucosa-associated bacterium in healthy subjects. ${ }^{42}$ The abundance of $A$. muciniphila was significantly reduced in patients with chronic inflammatory diseases, including obesity and Crohn's disease. The bacterium converts mucin to SCFA acetate and propionate, thus activating host epithelial cells to produce antimicrobial peptides such as defensins and Ctype lectin that play key roles in homeostasis. ${ }^{42,46}$

\section{Chronic urticaria}

Urticaria is commonly seen in dermatologic practice, clinically characterized by wheals, angioedema, or both. Of the $20 \%$ of individuals who experience acute urticaria, $0.1 \%$ will develop chronic symptoms, and most of them turn into chronic spontaneous urticaria (CSU). ${ }^{80}$ Chronic urticaria (CU) occurs when there are recurrent wheals that persist at a loss for six weeks. It divides into chronic spontaneous urticaria and chronic inducible urticaria (chronic physical urticaria). ${ }^{81,82}$ The pathogenesis of $\mathrm{CU}$ includes imbalances in immunity, inflammation, and coagulation. In $\mathrm{CU}$, when the antigen is entering the body its binds to the IgE high affinity (FceRIa) Fc receptor located on the mast cells and stimulates circulating basophils in the skin, which induce degranulation of mast cells. When the same antigen is encountered for the second time, these IgE antibodies that were already presented on the mast cells and basophils immediately bind to the antigen and develop an allergic reaction more quickly. ${ }^{81}$

$\mathrm{CU}$ is also triggered by autoimmunity. The relationship of gut microbiota affects CU's immune system has been studied in these past years. Some authors hypothesized that these immune changes in CU are also involved in gut microbiome dysbiosis. There was a significant difference in microbial composition in $\mathrm{CU}$ or CSU patients compared to healthy controls in all articles. $^{48-51}$ Enterobacteria, Bacteroidetes, Firmicutes, and Bifidobacteriales, could be markers for inflammatory diseases. All articles found a higher abundance of Enterobacteria in CU. Studies from Lu et al. in 2019 demonstrated a reduced number of Bacteroidetes and an increase in Proteobacteria. This result was following the research conducted by PolkowskaPruszynska in 2020 in other inflammatory diseases such as asthma and dermatitis atopic. ${ }^{83}$ The anti-inflammatory bacteria such as Akkermansia muciniphila, Faecalibacterium prausnitzii, Clostridium 
antibiotic treatments. ${ }^{19,21}$

Yan et al. in 2017 reported a decrease in Actinobacteria and increased Proteobacteria in patients with moderate and severe acne. They also reported that Bifidobacterium, Butyrocicoocus, Coprobacillus, Lactobacillus, and Allobaculum were decreased at the genus level. ${ }^{20}$ The Proteobacteria phylum has many pathogenic bacteria such as E. coli, Salmonella, and Vibrio cholerae. Overgrowth of Proteobacteria resulting from the host's inability to keep commensal Proteobacteria in a minor fraction and reduced resistance to colonization by the microbial community can further facilitate inflammation or invasion by exogenous pathogens. ${ }^{60}$ They contribute to the overgrowth of pathogen bacteria in the gastrointestinal, which results in proinflammatory cytokine stimulation and gastrointestinal dysfunction. Decreased levels of Actinobacteria and its genera turn into dysbiosis and induce proinflammatory cytokines leading to leaky gut.

Lactobacillus and Bifidobacterium are the most studied species of probiotics. Bifidobacterium and Lactobacillus induce regulatory dendritic cells and CD4+Foxp3+T cells (regulatory $\mathrm{T}$ cells), resulting in hyporesponsive $\mathrm{B}$ cells and $\mathrm{T}$ helper cells without apoptosis along with suppression of cytokines production. Thus, the less abundance of Bifidobacterium and Lactobacillus will result in cytokine production, including tumor necrosis factor- a and interferon-g. The reduction of these bacteria directly affects intestinal epithelial barrier function by decreasing intestinal permeability and enhancing abdominal epithelial resistance. ${ }^{61-63}$

\section{Atopic dermatitis}

Atopic dermatitis is a chronic relapsing eczematous rash, and it is often the first manifestation of the atopic march. ${ }^{64}$ As a chronic relapsing disorder, current treatment strategies must focus on controlling the symptoms rather than curing them. The clinical appearance of atopic dermatitis may vary depending on several factors, such as the dermatitis stage, the age of patients, skin color, and infection. Atopic dermatitis plaques may appear as blisters (acute), thickened skin with increased skin markings (chronic), or a mixture of both (subacute). The preference for clinical manifestation is varied in every span of age. At infancy, the lesions appear mainly on the face, neck, and extremities' extensor surface. Contrarily, the flexor surface of the extremity is a common clinical finding in childhood and adulthood. However, the clinical features of $\mathrm{AD}$ can be deconstructed into seven general categories, which are inflammation, infection, irritation, itch, ichthyosis (dry skin), immunological influences, and impeding comorbid conditions. ${ }^{64}$

Even though it is intriguing to focus on the skin when studying $\mathrm{AD}$ as the main pathology site, the complexity of immunological changes occurs. It connects with other organs like the gut and lungs.
Stimulatory antigens crossing these barriers can give rise to inappropriate immune stimulation. ${ }^{65}$ Recent evidence has revealed that dysbiosis microbiota in the human body is associated with AD. ${ }^{66}$ It has become increasingly evident that gut microbiota play a vital role in regulating innate and adaptive immunity and developing allergic diseases. ${ }^{67}$

The reduced intensity and diversity of microbes lead to abnormal immune maturation in early childhood. The lower diversity of microbiota could be related to a limited bacterial turnover, resulting in insufficient $\mathrm{T}$ helper (Th) 1 cell induction and the failure to suppress Th2 responses. The switching of the immune stimulation towards a pronounced Th2-phenotype is a major mechanism to maintain allergy development. ${ }^{30,35}$ However, there were discrepancies with the findings of our review articles. The disagreement was probably due to inter-ethnic variations, age, and first feeding time.

Five articles reported the increased abundance of Bacteroides spp., which are common inhabitants of the human gut. However, their increased presence has been associated with food allergies and other atopic manifestations. ${ }^{27,35-38}$ Indeed, higher levels of Bacteroides in atopy could lead to the continuous production of lipopolysaccharides (LPS), the major component of the gram-negative cell wall, in the gut, which could trigger an inflammatory response. Moreover, Bacteroides species were reported to alter gut permeability. ${ }^{35}$ Zeng et al. in 2016 reported a decrease of Bacteroides

Table 4. Articles discussing relationship between gut and skin microbiome in urticaria.

\begin{tabular}{lll} 
Types of studiy & \multicolumn{1}{c}{ CU patient vs. healthy control } & \\
& Increase & Decrease \\
Ten chronic urticaria (CU) patients compared with healthy & Proteobacteria & Bacteroidetes \\
controls of their fecal microbiota with 16SrRNA sequences. & Actinobacteria & Faecalibacterium, \\
They found a highly different microbial composition between & Enterobacteriales, & Prevotella, Lachnobacterium; \\
those two. & Lactobacilales, & Faecalibacterium prausnitzii, \\
& Pseudomonadales & Prevotella copri, \\
& Veillonella, & Bacteroides fragilis, \\
& Stterella, Streptococcus, & Bacteroides plebeius \\
& Clostridium, and Escherichia & \\
Twenty CU patients and 20 healthy individuals collected their & Enterobacteriaceae & Akkermansia muciniphilia, \\
fecal samples and samples are sequenced using bacterial PCR. & & Clostridium leptum, \\
The relative amounts of bacteria were different significantly. & & Faecalibacterium prausnitzii \\
The fecal sample of twenty CU patients and 20 match individuals & Bacteroidetes (p>0.05) & Lactobacillus; \\
were collected and analyzed their bacterial with PCR. & & Bifidobacterium
\end{tabular}

They demonstrated the relative amount of Lactobacillus and

Bifidobacterium were significantly higher in fecal samples

from controls to CU.

A hundred chronic spontaneous urticaria and 100 healthy individuals were analyzed their fecal samples and sequence by $16 \mathrm{~S}$ rRNA. The result shows alterations in gut microbes and metabolites.
Enterobacteriaceae Bacteroidetes (relative increase) Bacteroides

$\begin{array}{ll}\text { Firmicutes; Bifidobacteriales } & \text { Wang et al., } \\ \text { Faecalibacterium; } & 2020^{51} \\ \text { Bifidobacterium; Ruminococcaceae } & \end{array}$
Bifidobacterium; Ruminococcaceae 